\title{
A new standard: age and distance for the open cluster NGC 6791 from the eclipsing binary member V20 $0^{\star \star \star}$
}

\author{
F. Grundahl ${ }^{1}$, J. V. Clausen ${ }^{2}$, S. Hardis ${ }^{2}$, and S. Frandsen ${ }^{1}$ \\ 1 Department of Physics and Astronomy, Aarhus University, Ny Munkegade, 8000 Aarhus C, Denmark \\ e-mail: fgj@phys.au.dk \\ 2 Niels Bohr Institute, University of Copenhagen, Juliane Maries Vej 30, 2100 Copenhagen Ø, Denmark
}

Received 5 August 2008 / Accepted 30 September 2008

\begin{abstract}
Context. We wish to determine accurate ages for open clusters and use this, in conjunction with colour-magnitude diagrams, to constrain models of stellar structure and evolution.

Aims. The detached eclipsing binary V20 in the old, metal-rich $([\mathrm{Fe} / \mathrm{H}]=+0.40)$ open cluster NGC 6791 is studied in order to determine highly accurate masses and radii of its components. This allows the cluster age to be established with high precision, using isochrones in the mass-radius diagram.

Methods. We employ high-resolution UVES spectroscopy of V20 to determine the spectroscopic orbit and time-series V, I photometry to obtain the photometric elements.

Results. The masses and radii of the V20 components are found to be $1.074 \pm 0.008 M_{\odot}$ and $1.399 \pm 0.016 R_{\odot}$ (primary) and $0.827 \pm 0.004 M_{\odot}$ and $0.768 \pm 0.006 R_{\odot}$ (secondary). The primary is located almost exactly at the hottest point along the cluster isochrone, and the secondary is a $\sim 7$ times fainter main-sequence star. We determine an apparent cluster distance-modulus of $(m-$ $M)_{V}=13.46 \pm 0.10$ (average of primary and secondary). The cluster age is obtained from comparisons with theoretical isochrones in the mass-radius diagram. Using the isochrones from Victoria-Regina with $[\mathrm{Fe} / \mathrm{H}]=+0.37$ we find $7.7 \pm 0.5 \mathrm{Gyr}$, whereas the Yonsei-Yale $\left(Y^{2}\right)$ isochrones lead to $8.2 \pm 0.5 \mathrm{Gyr}$, and BaSTI isochrones to $9.0 \pm 0.5 \mathrm{Gyr}$. In a mass-radius diagram, the 7.7 Gyr VRSS and 9.0 Gyr BaSTI isochrones overlap nearly perfectly despite the age-difference. This model dependence, which is significantly larger than the precision determined from mass, radius, and abundance uncertainties, prevents a definitive age-determination of the cluster.

Conclusions. Using detached eclipsing binaries for determination of cluster ages, the dominant error is due to differences among stellar models and no longer to observational errors in cluster reddening and distance. By observing a suitable number of detached eclipsing binaries in several open clusters it should be possible to calibrate the age-scale and provide firm constraints which stellar models must reproduce.
\end{abstract}

Key words. stars: evolution - stars: binaries: spectroscopic - stars: binaries: eclipsing - techniques: photometric techniques: spectroscopic - galaxy: open clusters and associations: individual: NGC 6791

\section{Introduction}

Among the old open clusters, NGC 6791 holds a special place: it is one of the oldest, most massive, and most metal-rich (Origlia 2006; Carretta et al. 2007; Anthony-Twarog et al. 2007, hereafter ATTM07) clusters known. In addition to these features, the cluster contains a population of hot blue stars (Liebert et al. 1994; Landsman et al. 1998), a large population of white dwarfs extending to the end of the cooling sequence has been found with HST (Bedin et al. 2005; Bedin et al. 2008), and extensive photometric studies have revealed a large population of variable stars in the cluster field (Bruntt et al. 2003; Mochejska et al. 2002; De Marchi et al. 2007).

For these reasons, NGC 6791 has been the subject of a number of studies since the work by Kinman (1965), and yet there

\footnotetext{
* Based on observations carried out at Nordic Optical Telescope at La Palma and ESO's VLT/UVES ESO, Paranal, Chile (75.D-0206A, 77.D-0827A).

$\star \star$ Tables 11 and 12 are only available in electronic form at the CDS via anonymous ftp to cdsarc.u-strasbg.fr (130.79.128.5) or via http://cdsweb.u-strasbg.fr/cgi-bin/qcat?J/A+A/492/171
}

is still not agreement over its basic parameters. Given a distance of $\sim 4 \mathrm{kpc}$ and a non-negligible reddening, determination of the cluster age is a complex problem when attempting to use the colour-magnitude diagram (hereafter CMD). Even with recent attempts to detect exo-planet transits (Bruntt et al. 2003; Mochejska et al. 2005; Montalto et al. 2007) - which has led to a substantial body of well calibrated photometry and a highprecision CMD (Stetson et al. 2003, hereafter SBG03) - it has proven very difficult to determine a precise cluster age. This is mainly related to the difficulty of obtaining precise reddening and distance estimates (see ATTM07 for an extensive discussion) and to the problem of transforming model isochrones to observed colours and magnitudes at the high metallicity of the cluster (Tripicco et al. 1995).

The range of ages proposed for NGC 6791 has extremes of 7 and $12 \mathrm{Gyr}$, but has in recent years seemed to converge on a value near 8 Gyr. This would put the cluster turnoff mass close to $1 M_{\odot}$ and thus bridge the "gap" between the turnoff mass of globular clusters (typically $0.8 M_{\odot}$ ) and younger open clusters, where convective overshoot has a marked effect on their turnoff morphology in the CMD. Such a high cluster age would also seem 
to suggest that atomic diffusion might have had sufficient time to act in its stars, such as found in the globular cluster NGC 6397 (Korn et al. 2006).

In the course of a study to try and detect exoplanetary transits in the cluster (Bruntt et al. 2003), we realized that the detached eclipsing system V20 (Rucinski et al. 1996) might offer the possibility to determine the masses and radii for stars near the turnoff. Rough estimates of the system parameters (Bruntt et al. 2003) suggested that although V20 is only at $V=17.34$, it would still be within the capabilities of UVES (Dekker et al. 2000) at the ESO VLT to measure precise radial velocites for its components.

It is well known that detached eclipsing binaries offer the possibility to determine precise masses and radii for the system components. If one or both components is close to its turnoff mass (for the age of the binary), it is possible to put tight constraints on the age of the system through a comparison of the position of the primary and secondary in a mass-radius $(M-R)$ diagram to theoretical isochrones. For stellar clusters, such an analysis has some significant advantages: the determination of the masses and radii is essentially independent of the usual "trouble-makers" such as reddening, distance, metallicity. Furthermore, since the comparison to models is carried out in the $M-R$ diagram, one avoids the difficult process of transforming the effective temperatures and luminosities of the models to observed colours and magnitudes. Thus, a determination of cluster ages in the $M-R$ diagram is essentially "the closest" one can get to a "direct" confrontation between observations and models.

In this paper we undertake a full analysis of the detached eclipsing binary system V20 in NGC 6791 and determine accurate values for the masses and radii of its components. This is used to determine a precise cluster age and to compare the ages derived using commonly used isochrones from three sources. Our main conclusion is that using such binary systems as V20, it is the "accuracy" of the available theoretical stellar models, which limit the obtainable age precision, and not the observational data.

\section{Photometry of V20}

The photometric data for V20 consists of $V$ (Johnson) and $I$ (Cousins) CCD observations from the $2.56 \mathrm{~m}$ Nordic Optical Telescope (NOT) and its stand-by camera StanCam. We refer to the telescope homepage for further information ${ }^{1}$. V20 was observed on 9 nights between April 2003 and July 2004, and a total of 298 and 300 exposures were obtained in $V$ and $I$, respectively. Primary eclipse observations were done on three nights, secondary eclipse observations on four nights, and out-of-eclipse phases on two nights. Although complete coverage of the light curve has not been secured outside eclipses, the data are sufficient for a detailed photometric analysis.

For all observations we employed an exposure time of $240 \mathrm{~s}$ in $V$ and $180 \mathrm{~s}$ in $I$. Seeing conditions varied substantially from night to night, with median values of $11^{\prime \prime} 00$ and $0 . ' 82$ in $V$ and $I$, respectively. The best seeing frames have a FWHM of 0.60 and 0 '. 46, respectively.

The bias frames and flat fields, used in the data reduction, were obtained during evening twilight on each observing night. All photometry was carried out with DAOPHOT/ALLSTAR/ALLFRAME (Stetson 1987, 1994) and DAOGROW (Stetson 1990) and transformed to a common coordinate system using MATCH and MASTER (Stetson, private

\footnotetext{
${ }^{1}$ http://www.not.iac.es
}
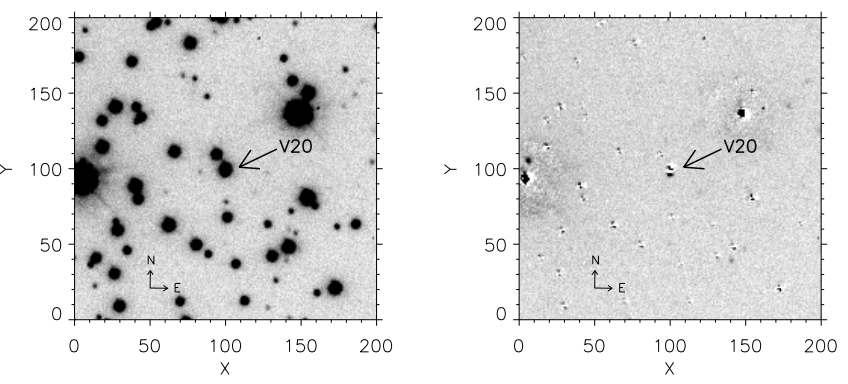

Fig. 1. The field of V20 in NGC 6791, centred at $(\alpha, \delta)_{2000}=$ $\left(19^{\mathrm{h}} 20^{\mathrm{m}} 54.30^{\mathrm{s}}+37^{\mathrm{d}} 45^{\mathrm{m}} 34.7^{\mathrm{s}}\right)$. Note, that in the left image V20 appears slightly elongated in the N-S direction, the FWHM in this $I$ band image is $0 !$ '46. The righthand image shows the star subtracted version of the left image. Note the larger than expected residuals at the position of V20, consistent with a fainter third light component. The pixel size is $0{ }^{\prime} 1755$ and the greyscale is identical for the two images.

comm.). For each frame we produced a point-spread function (PSF) using the brightest stars in the field, and subsequently carried out aperture photometry in large apertures (neighbour stars subtracted) using the NEDA routine provided with DAOPHOT. Subsequently the aperture photometry was fed into DAOGROW to obtain the final large-aperture magnitudes. We found that this prodedure gives slightly better photometric precision than using profile-fitting photometry.

During the reductions of the data, we noticed on the best seeing frames $\left(F W H M=0 .{ }^{\prime} 46\right)$ that V20 appeared slightly elongated, and we interpret this as a signature of a third light component due to a chance alignment with a cluster star. This has to be properly taken into account in the final analysis of the lightcurves. In Fig. 1 we show the field in NGC 6791 centred on V20. Attempts were made to carry out ALLFRAME photometry with two components at the position of V20 separated by $0 . ' 4$. This improved the residuals in the fit for the best seeing frames, but the large seeing variations did not allow a sufficient precision in the magnitudes of the V20 primary+secondary and the third light component. We therefore include the light from the third star in the light curves, and deal with it in the subsequent analyses.

\subsection{Light curves and standard indices}

With the instrumental photometry in hand, we proceeded to transform the observations to the $V$ and $I$ standard system. In SBG03 a large effort was put into this transformation, and we have used the available photometry from this source as internal standard stars. For each frame, a linear transformation from instrumental magnitudes to standard magnitudes, using $(V-I)$ as colour term, was calculated, and subsequently we averaged the coefficient for the colour term for all frames (for a given filter) and used this for the final determination of the zeropoint for each frame. Our photometry is therefore on the same system as SBG03 with the out-of-eclipse magnitudes given by their photometry as listed in Table 1 . The accuracy of the photometry is in the range 0.01 to 0.02 as mentioned in SBG03.

The light curves for V20 are listed in Tables 11 and 12 and are shown in Figs. 2 and 3 with phases calculated from the ephemeris given in Eq. (1). V20 is clearly well detached with practically constant light level outside eclipses. Secondary eclipse occurs at phase 0.50 , and the eclipses are of the same duration, supporting that the orbit is circular. As seen from the different eclipse depths, V20 consists of two stars of rather 
Table 1. Standard V,I photometry for V20 outside eclipses from SBG03; V20 is star number 8600 in their list. The mean values, with errors, are based on $925(V)$ and $703(I)$ observations, respectively. Photometry for the two components and the 3rd star was calculated from the depths of the total secondary eclipse, assuming that all three stars are located on the main sequence of NGC 6791; see text for details.

\begin{tabular}{lrrr}
\hline \hline Object & $V$ & $I$ & $V-I$ \\
\hline V20 + companion (SBG03) & 17.3390 & 16.3560 & 0.9830 \\
& \pm 5 & \pm 6 & \pm 8 \\
Primary & 17.6400 & 16.7277 & 0.9123 \\
Secondary & 19.8686 & 18.6353 & 1.2333 \\
3rd star & 19.4322 & 18.2911 & 1.1322 \\
\hline
\end{tabular}
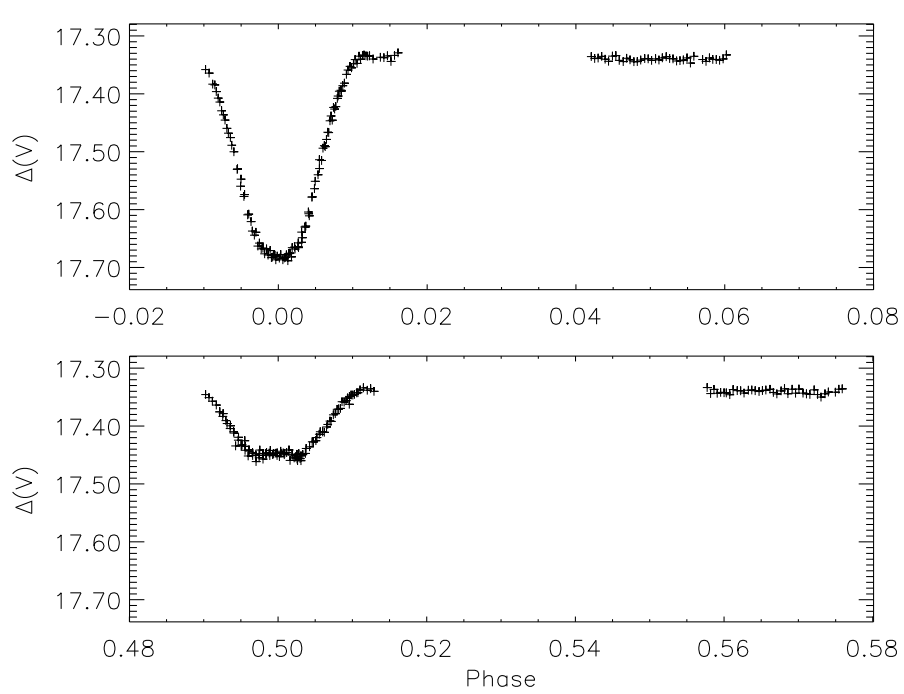

Fig. 2. $V$ light curve for V20 (third star included). Only the observed phase intervals are shown.

different surface fluxes. Secondary eclipse is total; depths are 0 . $111(V)$ and $0.142(I)$. The close companion is included in the light curves, meaning that a significant amount of third light is present.

Throughout the paper, the component eclipsed at the deeper eclipse at phase 0.0 is referred to as the primary (p), and the other as the secondary (s) component.

Standard, out-of-eclipse, V,I photometry for V20 is listed in Table 1 together with calculated individual photometry for the three stars. In order to obtain constraints on the contribution from the third light, we shall attempt to determine its value from the available light curve.

We first determined the depth of the total secondary eclipse in $V$ and $I$ to be $0^{\mathrm{m}} 111$ and $0^{\mathrm{m}} 142$, respectively. Since this represents the case where the secondary component of the binary is totally eclipsed, we can determine the magnitudes for the secondary component, and for the sum of the primary and third light component. If we assume (as is verified later from spectroscopy) that V20 is a member of the cluster, and that the third light is also a cluster member, then by assuming that it is located on the main sequence, a unique combination can be calculated, for which the sum of primary and third light matches the light during total eclipse. In practical terms, we fitted a low order polynomial to the main-sequence of the cluster (representing possible locations for the third light component). Then, for each point along the polynomial, the corresponding location of the primary component was calculated, such that the sum of their light matched the location of the light during total eclipse of the secondary component.
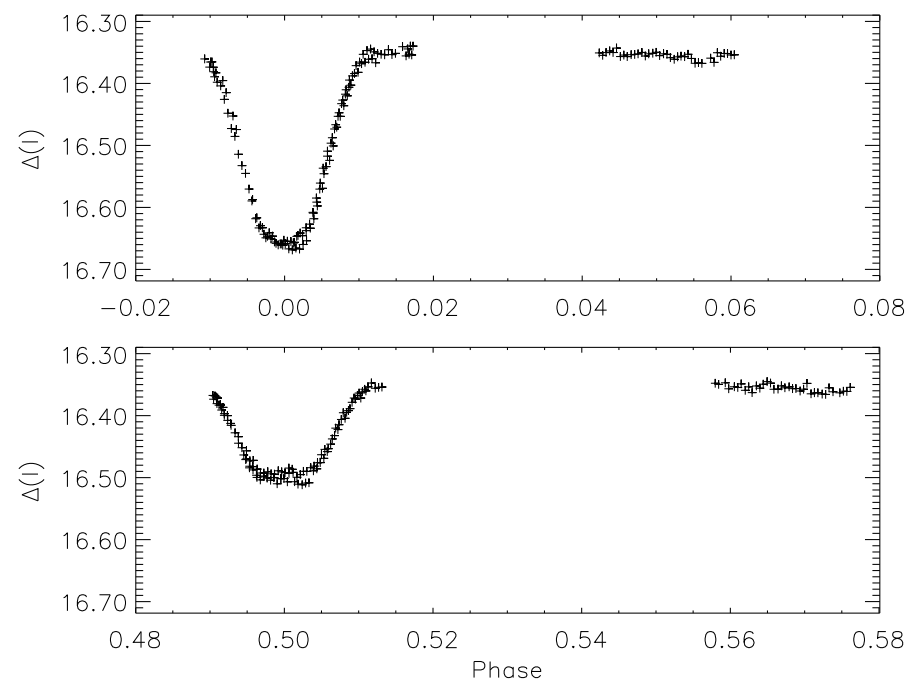

Fig. 3. I light curve for V20 (third star included). Only the observed phase intervals are shown.

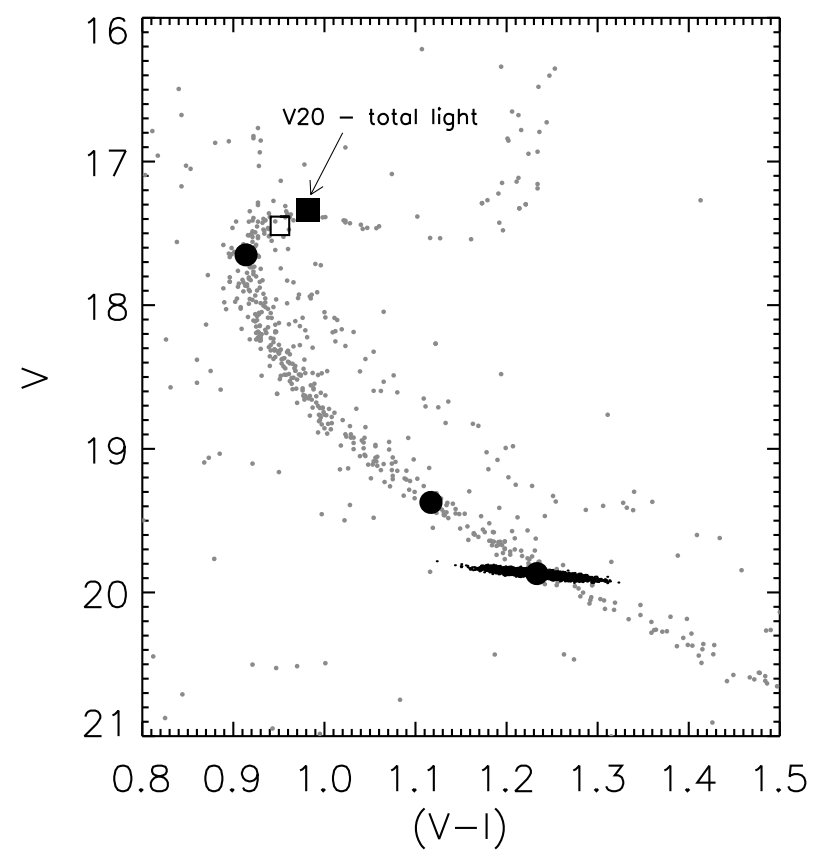

Fig. 4. The $(V-I, V)$ colour-magnitude diagram for NGC 6791 with V20 and its components overplotted. The filled square indicates the location of the total light of the entire system. The light of the primary and third component (light during total eclipse of the secondary) is indicated by an open square. In order of decreasing luminosity, the filled circles indicate the location of the primary, the third star, and the secondary. For the secondary, we have overplotted the possible locations if errors of 0.002 in $V$ and $I$-depths of the total eclipse are assumed.

The results of this exercise is given in Table 1 for the primary, secondary and third star. In Fig. 4 we show the cluster $(V-I), V$ colour-magnitude diagram based on the Stetson et al. (2003) photometry with the location of the V20 combined light and the individual components indicated. We have attempted to estimate the uncertainty in the location of the secondary component by assuming that the uncertainty in the estimate of the $V$ and $I$ depths of the total eclipse is 0.002 and then calculating its position 10000 times at random from a normal distribution of $\sigma=0.002$ in each colour and calculating the position of the secondary. As can be seen from Fig. 4 it appears that three 
Table 2. Times of minima for V20. O-C values are calculated for the ephemeris given in Eq. (1) adopting a circular orbit. References are: M2002, Mochejska et al. (2002); B2003, Bruntt et al. (2003); G2008, this paper.

\begin{tabular}{lllrll}
\hline \hline $\begin{array}{l}\text { HJD } \\
-2400000\end{array}$ & rms & Type & O-C & Band & Ref. \\
\hline 52109.77415 & 0.00110 & $\mathrm{P}$ & 0.00215 & $R$ & M2002 \\
53151.60485 & 0.00030 & $\mathrm{P}$ & -0.00161 & $V$ & G2008 \\
53151.60449 & 0.00100 & $\mathrm{P}$ & -0.00125 & $I$ & - \\
53180.54732 & 0.00060 & $\mathrm{P}$ & 0.00153 & $V$ & - \\
53180.54747 & 0.00070 & $\mathrm{P}$ & 0.00138 & $I$ & - \\
52102.53467 & 0.00100 & $\mathrm{~S}$ & -0.00373 & $V$ & $\mathrm{~B} 2003$ \\
52102.53332 & 0.00080 & $\mathrm{~S}$ & -0.00238 & $V$ & - \\
52811.56249 & 0.00100 & $\mathrm{~S}$ & 0.00101 & $V$ & $\mathrm{G} 2008$ \\
52811.56404 & 0.00090 & $\mathrm{~S}$ & -0.00054 & $I$ & - \\
52869.44066 & 0.00210 & $\mathrm{~S}$ & 0.00039 & $V$ & - \\
52869.44309 & 0.00170 & $\mathrm{~S}$ & -0.00204 & $I$ & - \\
\hline
\end{tabular}

cluster stars can indeed match the observed total light (as expected) and that the third light component has a luminosity in between the primary and secondary.

The exact luminosity for the third star is somewhat dependent on how the fiducial for the main-sequence is derived. We estimate that the uncertainty in its determined position is not larger than $0 .{ }^{\mathrm{m}} 15$ - this is based on experiments where small shifts in colour were added to the fitted main-sequence polynomial, while it still appeared to represent the main-sequence. We note, that high-resolution imaging would allow a direct determination of the contribution to the total light from the third star. For the expected separation $\left.(\sim 0)^{\prime} 4\right)$, this would probably require adaptive optics.

\subsection{Times of minima and ephemeris}

From the $V$ and $I$ light curve observations, two times of minima per band have been derived for both eclipses. They are listed in Table 2 together with times redetermined from the photometry by Bruntt et al. (2003) and Mochejska et al. (2002). The method of Kwee \& van Woerden (1956) was applied throughout.

Weighted linear least square fits to the times of minima yield formally identical periods of $14.46989 \pm 0.00005$ and $14.46999 \pm 0.00017$ for primary and secondary eclipses, respectively. Since only three times per eclipse are available, we have independently determined both the epoch and the period from JKTEBOP analyses of our $V$ and $I$ light curves; see Sect. 2.3. The periods obtained from the two light curves agree well and are close to those given above. We adopt the following linear ephemeris for all analyses in this paper:

$$
\text { Min I }=\begin{array}{r}
2453151.6061 \\
\pm 9
\end{array} \quad \begin{array}{r}
14.469918 \\
\pm 25
\end{array} \quad \times E
$$

\subsection{Photometric elements}

Since V20 is well detached with relative component radii of only about 0.045 and 0.025 , respectively, we have adopted the simple Nelson-Davis-Etzel model (Nelson \& Davis 1972; Etzel 1981; Martynov 1973) for the light curve analyses. It represents the deformed stars as biaxial ellipsoids and applies a simple bolometric reflection model. We have used the corresponding JKTEBOP ${ }^{2}$ code, which is a revised and extended version of the original

${ }^{2}$ http://www.astro.keele.ac.uk/ jkt/
EBOP code (Etzel 1981). The Levenberg-Marquardt minimization algorithm (MRQMIN: Press et al. 1992) is used for the lestsquares optimization of the parameters, and the code has been extended to include also non-linear limb darkening and adjustment of epoch and orbital period. In one of its modes, JKTEBOP performs Monte Carlo simulations, which we use to assign realistic errors to the photometric elements. For further information, we refer to e.g. Southworth et al. $(2004 b ; 2007)$ and Bruntt et al. (2006).

The $V$ and $I$ light curves were analysed independently, with equal weights assigned to all observations. The magnitude at quadrature was always included as an adjustable parameter, and the phase of primary eclipse was allowed to shift from 0.0. In initial JKTEBOP analyses, the epoch and orbital period was included as free parameters and then fixed at the values given in Eq. (1); see Sect. 2.2. A circular orbit was assumed throughout, and the mass ratio between the components was kept at the spectroscopic value (Table 8). Gravity darkening coefficients corresponding to convective atmospheres were applied, and the simple bolometric reflection model built into EBOP/JKTEBOP was used. Linear limb darkening coefficients were either assigned from theoretical calculations (Van Hamme 1993; Claret 2000) according to the effective temperatures, surface gravities, and abundance, or left free.

In text and tables on photometric solutions we use the following symbols: $i$ orbital inclination; $r$ relative radius; $k=r_{\mathrm{s}} / r_{\mathrm{p}}$; $u$ linear limb darkening coefficient; $y$ gravity darkening coefficient; $J$ central surface brightness ratio; $L$ luminosity; $l_{3}$ third light fraction.

As mentioned in Sects. 2 and 2.1, a close star is included in the light curve data. The corresponding amount of third light $l_{3}=L_{3} /\left(L_{\mathrm{p}}+L_{\mathrm{s}}+L_{3}\right)$, as calculated from the information in Table 1 , is $0.146 \pm 0.021(V)$ and $0.168 \pm 0.024(I)$. Uncertainties are based on realistic estimates of the accuracy of the magnitudes of the total light $( \pm 0.010)$, the depths of secondary eclipse $( \pm 0.002)$, and the magnitudes calculated for the companion $( \pm 0.15)$. We have adopted these $l 3$ results, which are based on the realistic assumption that the third star is a main sequence cluster member, since it turns out that the amount of third light is not well constrained by the light curves; see below.

The photometric solutions for different adopted theoretical linear limb darkening coefficients are given in Table 3, and (O-C) residuals are shown in Figs. 5 and 6, which clearly reveal the higher quality of the $V$ light curve. Except for a larger scatter, partly due to small nightly differences, there are no serious systematic trends in the $I$ residuals. As seen, the orbital inclination is close to $90^{\circ}$, and the elements of the individual solutions agree well. The two quite different components are small compared to the radius of the orbit; for both components the individual radii obtained agree within about $\pm 1 \%$. Additional tests reveal that the ratio of relative radii $k$ is well constrained by the light curves.

Including the linear limb darkening coefficients for both components as adjustable parameters was only partly successful, since those for the secondary components converged towards unrealistically low and also very uncertain values. For the primary component, the $V$ value is close to that by Van Hamme (1993), whereas the $I$ value is just between the two theoretical coefficients. They are almost independent of whether $u_{\mathrm{s}}$ is fixed at the theoretical values or adjusted.

Adopting a non-linear limb darkening law (square root) was also attempted but has no significant influence on the solutions, as also found by Lacy et al. (2008). 
Table 3. Photometric solutions for V20 from the JKTEBOP code. A photometric scale factor (the magnitude at quadrature) and the phase of primary eclipse were included as free parameters. Linear limb darkening coefficients from Claret (2000), van Hamme (1993), and free, respectively. The errors quoted for the adjusted parameters are the formal errors determined from the iterative least squares solution procedure.

\begin{tabular}{lrrrrrr}
\hline \hline $\begin{array}{l}\text { Band } \\
\text { Limb } \\
\text { darkening }\end{array}$ & $\begin{array}{r}V \\
\text { C00 }\end{array}$ & $\begin{array}{r}V H 93 \\
\text { VHree }\end{array}$ & $\begin{array}{r}V \\
\text { C00 }\end{array}$ & $\begin{array}{r}I \\
\text { VH93 }\end{array}$ & $\begin{array}{r}I \\
\text { Free }\end{array}$ \\
\hline$i\left(^{\circ}\right)$ & 89.76 & 89.74 & 89.85 & 89.78 & 89.78 & 89.86 \\
& \pm 11 & \pm 9 & \pm 17 & \pm 16 & \pm 15 & \pm 28 \\
$r_{\mathrm{p}}+r_{\mathrm{s}}$ & 0.0703 & 0.0695 & 0.0696 & 0.0709 & 0.0703 & 0.0705 \\
$k=r_{\mathrm{s}} / r_{\mathrm{p}}$ & 0.539 & 0.548 & 0.545 & 0.544 & 0.553 & 0.547 \\
& \pm 1 & \pm 1 & \pm 3 & \pm 2 & \pm 2 & \pm 4 \\
$r_{\mathrm{p}}$ & 0.0457 & 0.0449 & 0.0450 & 0.0459 & 0.0454 & 0.0456 \\
$r_{\mathrm{s}}$ & 0.0246 & 0.0246 & 0.0245 & 0.0250 & 0.0250 & 0.0249 \\
$u_{\mathrm{p}}$ & 0.72 & 0.61 & 0.65 & 0.56 & 0.44 & 0.51 \\
& & & \pm 3 & & & \pm 4 \\
$u_{\mathrm{s}}$ & 0.79 & 0.74 & 0.34 & 0.62 & 0.54 & 0.43 \\
& & & \pm 25 & & & \pm 26 \\
$y_{\mathrm{p}}$ & 0.39 & 0.39 & 0.39 & 0.25 & 0.25 & 0.25 \\
$y_{\mathrm{p}}$ & 0.46 & 0.46 & 0.46 & 0.29 & 0.29 & 0.29 \\
$J_{\mathrm{s}} / J_{\mathrm{p}}$ & 0.448 & 0.447 & 0.381 & 0.599 & 0.594 & 0.562 \\
$L_{\mathrm{s}} / L_{\mathrm{p}}$ & \pm 3 & \pm 3 & \pm 35 & \pm 5 & \pm 4 & \pm 56 \\
$l_{3}$ & 0.126 & 0.127 & 0.127 & 0.173 & 0.174 & 0.174 \\
$\sigma(\mathrm{mag})$ & 0.146 & 0.146 & 0.146 & 0.168 & 0.168 & 0.168 \\
\hline
\end{tabular}
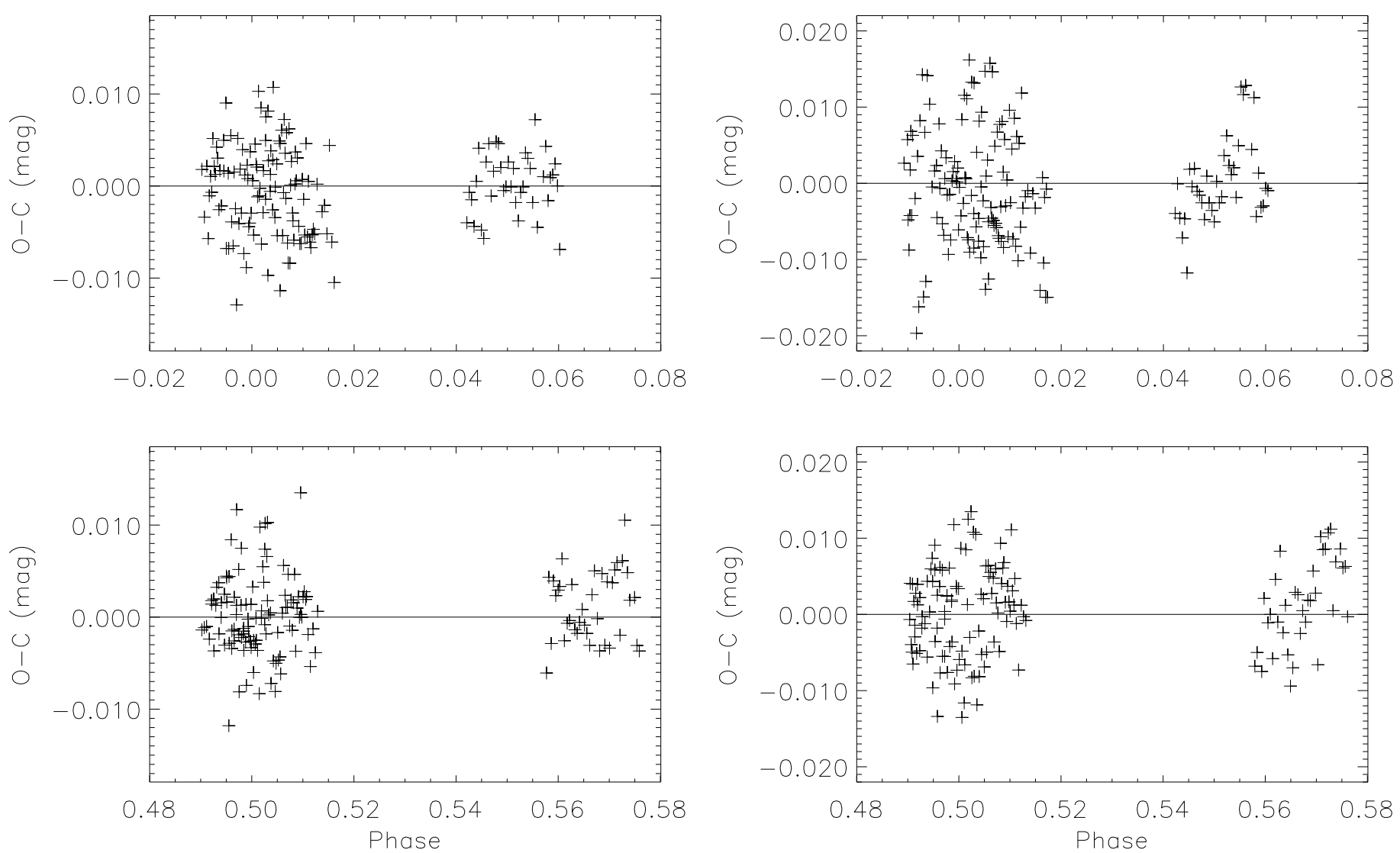

Fig. 5. (O-C) residuals of the $V$ observations from the theoretical light curves for the VH93 parameters listed in Table 3.

As already mentioned the amount of third light is not well constrained by the light curves themselves. If included as free parameters, much too low but also very uncertain values are obtained $(V: 0.04 \pm 0.08 ; I: 0.13 \pm 0.10)$. Upper third light limits, leading to an orbital inclination of $90^{\circ}$, are about $0.16(\mathrm{~V})$

Fig. 6. (O-C) residuals of the $I$ observations from the theoretical light curves for the VH93 parameters listed in Table 3.

and $0.18(I)$. The effect on the photometric elements of changing the adopted $l_{3}$ values by $15 \%$, i.e. by about their uncertainties given above, is shown in Table 4.

The final, adopted photometric elements for V20 are listed in Table 5. Higher weight has been given to the results based 
Table 4. The effect of changing the amount of third light $l_{3}$ by $15 \%$. Photometric solutions for V20 from the JKTEBOP code. A photometric scale factor (the magnitude at quadrature) and the phase of primary eclipse were included as free parameters. Linear limb darkening coefficients from van Hamme (1993) were adopted, see Table 3. The errors quoted for the adjusted parameters are the formal errors determined from the iterative least squares solution procedure.

\begin{tabular}{lrr}
\hline \hline Band & $V$ & $I$ \\
\hline$i\left(^{\circ}\right)$ & 89.63 & 89.62 \\
& \pm 6 & \pm 9 \\
$r_{\mathrm{p}}+r_{\mathrm{s}}$ & 0.0697 & 0.0704 \\
& \pm 3 & \pm 5 \\
$k=r_{\mathrm{s}} / r_{\mathrm{p}}$ & 0.540 & 0.542 \\
& \pm 1 & \pm 2 \\
$r_{\mathrm{p}}$ & 0.0453 & 0.0457 \\
$r_{\mathrm{s}}$ & 0.0244 & 0.0248 \\
$J_{\mathrm{s}} / J_{\mathrm{p}}$ & 0.446 & 0.590 \\
$L_{\mathrm{s}} / L_{\mathrm{p}}$ & \pm 3 & \pm 4 \\
$l_{3}$ & 0.123 & 0.168 \\
$\sigma(\mathrm{mag})$ & 0.124 & 0.143 \\
& 0.0044 & 0.0067 \\
\hline
\end{tabular}

Table 5. Adopted photometric elements for V20. The individual flux and luminosity ratios are based on the mean stellar and orbital parameters.

\begin{tabular}{|c|c|c|}
\hline$i\left({ }^{\circ}\right)$ & \multicolumn{2}{|c|}{$89.76 \pm 0.15$} \\
\hline$r_{\mathrm{p}}$ & \multicolumn{2}{|c|}{$0.0452 \pm 0.0005$} \\
\hline$r_{\mathrm{s}}$ & \multicolumn{2}{|c|}{$0.0248 \pm 0.0002$} \\
\hline & $V$ & I \\
\hline$J_{\mathrm{s}} / J_{\mathrm{p}}$ & $\begin{array}{r}0.451 \\
+7\end{array}$ & $\begin{array}{r}0.590 \\
+8\end{array}$ \\
\hline$L_{\mathrm{s}} / L_{\mathrm{p}}$ & $\begin{array}{r}0.129 \\
\pm 4\end{array}$ & $\begin{array}{r}0.172 \\
\pm 6\end{array}$ \\
\hline
\end{tabular}

on Van Hamme (1993) linear limb darkening coefficients. Uncertainties are based on interagreement between the $V$ and $I$ solutions, the uncertainties of the amount of third light, and Monte Carlo simulations.

\section{Spectroscopy for V20}

The spectroscopic observations for V20 were carried out in service mode with UVES at the ESO VLT during allocation periods 75 and 77 . Since V20 is at declination $+37^{\circ}$, it can on Paranal only be observed at an airmass larger than 2.1 - therefore all observations were carried out near meridian passage. Due to the faintness of V20, and in order to minimize slit losses, a slit of 1 .'20 width, corresponding to a resolution of approximately 37000 , was used. The slit was aligned along the parallactic angle (ELEV mode), and the ADC was not inserted in the beam, since it causes a slight loss of flux. The standard $580 \mathrm{~nm}$ setup, and on-chip binning of $2 \times 2$ pixels, was used for all observations. The corresponding wavelength ranges covered at the two CCD detectors employed in UVES are approximately 4775-5750 $\AA$ and 5875-6830 , respectively. A total of 17 epochs were obtained, see Table 6 , each with a ThAr exposure attached. The FWHM from the image headers for the start and end of each exposure are included. The typical $\mathrm{S} / \mathrm{N}$ per pixel for the red chip was between 15 and 25; two of the spectra had to be omitted in the data analysis due to a very low signal. After careful check of the wavelength calibrations, we decided to apply the pipeline reduced spectra for the analyses described below.
Table 6. Log of spectroscopic observations for V20 obtained with UVES, ordered by orbital phase. The heliocentric Date (HJD) is given at mid-exposure, exposure time $\left(T_{\exp }\right)$ in seconds, and seeing (FWHM) in arcseconds. Observations excluded in the analyses are marked by *.

\begin{tabular}{lcccc}
\hline \hline HJD & Phase & $T_{\exp }$ & Airmass & $F W H M$ \\
\hline 2453847.88977 & 0.11939 & 4060 & $2.34-2.15$ & $0.73-0.66$ \\
2453573.65870 & 0.16759 & 2700 & $2.20-2.15$ & $0.79-0.69$ \\
2453588.62071 & 0.20160 & 2700 & $2.19-2.15$ & $0.89-0.68$ \\
2453588.65279 & 0.20381 & 2700 & $2.15-2.25$ & $0.67-0.60$ \\
2453589.60199 & 0.26941 & 2700 & $2.27-2.16$ & $0.82-0.83$ \\
2453589.63401 & 0.27162 & 2700 & $2.16-2.18$ & $0.96-0.75$ \\
2453590.62070 & 0.33981 & 2700 & $2.18-2.16$ & $0.50-0.73$ \\
2453590.65270 & 0.34202 & 2700 & $2.16-2.28$ & $0.64-0.63$ \\
2453880.81608 & 0.39489 & 4060 & $2.24-2.16$ & $0.72-$ \\
2453623.51512 & 0.61311 & 2700 & $2.23-2.15$ & $0.87-0.87$ \\
2453623.54710 & 0.61532 & 2700 & $2.15-2.21$ & $0.98-0.81$ \\
$2453986.50872 *$ & 0.69919 & 4060 & $2.37-2.15$ & -1.11 \\
$2453957.58875 *$ & 0.70057 & 4060 & $2.37-2.15$ & $1.39-1.95$ \\
2453943.67176 & 0.73878 & 4060 & $2.16-2.24$ & $0.84-0.87$ \\
2453871.82770 & 0.77372 & 4060 & $2.32-2.15$ & $0.45-0.36$ \\
2453626.50659 & 0.81985 & 2700 & $2.23-2.15$ & $0.78-0.72$ \\
2454003.51981 & 0.87481 & 4060 & $2.15-2.33$ & $0.93-0.81$ \\
\hline
\end{tabular}

\subsection{Radial velocities and spectroscopic elements}

In order to reduce/eliminate possible systematic velocity errors, we have decided to apply the broadening function (BF) formalism (Rucinski 1999, 2002, 2004; with useful IDL routines and a cookbook from his homepage ${ }^{3}$ ) for radial velocity measurements of V20. Based on results reported in the literature, we expected this method to be well suited for the analysis of the sharp lined but otherwise non-trivial spectra of V20 with three set of lines. As seen below, our expectations have been fully met.

The BF method applies only one (unbroadened) template at a time, so we have decided to perform several analyses adopting three different synthetic templates, corresponding to each of the three set of lines present in the V20 spectra. For calculation of templates we have used the bssynth tool (Bruntt, private communication), which applies the SYNTH software (Valenti \& Piskunov 1996) and modified ATLAS9 models (Heiter 2002). The UVES spectra were first carefully cleaned and normalized, and then, like the template spectra, logarithmically rebinned to a constant velocity step of $1.0 \mathrm{~km} \mathrm{~s}^{-1}$.

The two spectral regions were analysed separately, and the position of the BF's, defining the radial velocities of the stars, were calculated by fitting Gaussians to the smoothed functions. A sample BF is shown in Fig. 7.

Spectroscopic elements were derived from the measured radial velocities using the method of Lehman-Filhés implemented in the SBOP program (Etzel 2004). The orbital period given in Eq. (1) was adopted, and the orbit was defined to be circular. Both double-lined and single lined solutions were done. Assuming that the radial velocity of the third component is constant, small shifts corresponding to the difference between its mean velocity and the individual values were added to measured velocities of the primary and secondary components. The shifts do not alter the derived system velocities and semi-amplitudes significantly but improve their uncertainties.

The orbital elements determined from the different spectral ranges using different templates are given in Table 7 together with the mean radial velocities for the third star. As seen, very accurate velocity semi-amplitudes are obtained; in general, the

3 http://www.astro.utoronto.ca/ rucinski/ 


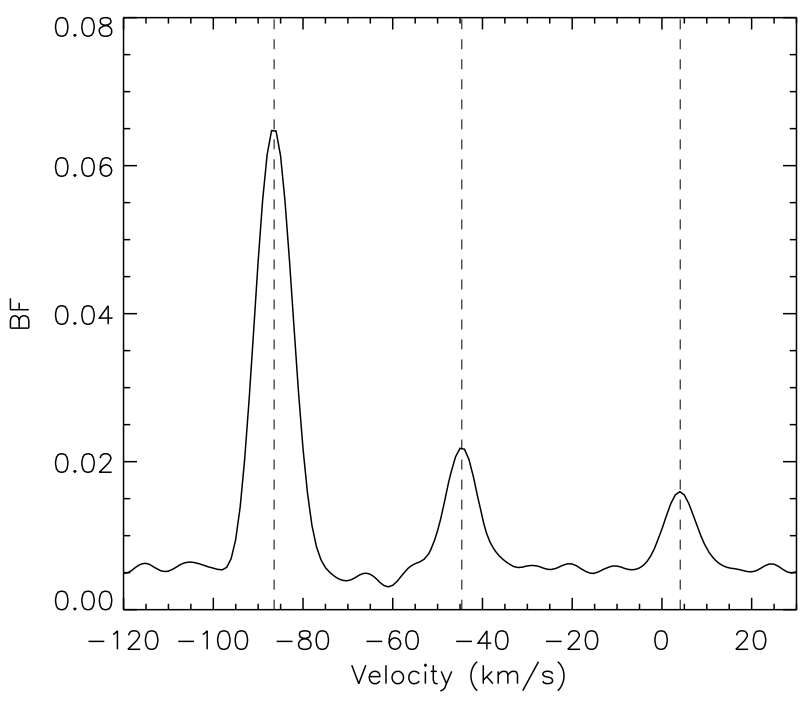

Fig. 7. Broadening function obtained for the $6100-6700 \AA$ region using the primary $(\mathrm{P})$ template. The UVES spectrum was taken at phase 0.342 , at $\mathrm{HJD}=2453590.65270$. From left to right the components are: primary, third star, secondary.

highest accuracy is reached for the 6100-6700 A region; Fig. 8 illustrates one of the solutions. The double-lined solutions and the single-lined solutions from the 6100-6700 A region agree extremely well, whereas the semi-amplitudes of the single-lined solutions from the 5100-5600 A region are systematically lower by about $0.10 \mathrm{~km} \mathrm{~s}^{-1}$. The primary and secondary single-lined solutions, except the secondary for the 5100-5600 ̊ region, give slightly different values for the system velocity, although the results formally agree within the errors.

In order to check for possible systematic velocity errors, we have analysed synthetic spectra calculated for the observed orbital phases. The three templates, broadened to $v \sin i$ values matching the observations, were shifted to their velocities at a given phase and combined according to the relative luminosities of the V20 components and the third star. BF analyses of the synthetic spectra show that the measured radial velocities deviate only slightly from the input values, typically below $\pm 0.15 \mathrm{~km} \mathrm{~s}^{-1}$ for the primary and $\pm 0.30 \mathrm{~km} \mathrm{~s}^{-1}$ for the secondary. Furthermore, adding the small shifts to the radial velocities measured from the observed spectra does not lead to significant changes of the orbital elements and their uncertainties. Two examples (marked by $*$ ) are included in Table 7 .

We adopt the weighted mean of the uncorrected single-lined solutions as the final spectroscopic elements for V20 listed in Table 8. Errors typical for the individual solutions have conservatively been assigned to the semi-amplitudes and the system velocity. As seen, minimum masses accurate to $0.7 \%$ and $0.5 \%$ have been obtained for the primary and secondary components, respectively. For comparison, masses derived from the corrected single-lined solutions are only $0.2 \%$ higher for the primary and $0.2 \%$ lower for the secondary component.

We have determined a systemic velocity for V20 of $-46.63 \pm$ $0.13 \mathrm{~km} \mathrm{~s}^{-1}$ (Table 8). This is in excellent agreement with the value of $-47.1 \pm 0.8 \mathrm{~km} \mathrm{~s}^{-1}$ as determined from 15 cluster members by Carraro et al. (2006) who also found the dispersion in the radial velocities of the 15 stars to be $2.2 \pm 0.4 \mathrm{~km} \mathrm{~s}^{-1}$. This leaves little doubt that the system is indeed a member of NGC 6791.

\section{Absolute dimensions and distance for V20}

Absolute dimensions for the components of V20 are calculated from the elements given in Tables 5 and 8. As seen in Table 9, masses and radii have been obtained to an accuracy of about $0.6 \%$ and $1.0 \%$, respectively. Individual $V, I, V-I$ magnitudes and indices are included, as calculated from the combined photometry (Table 1) and the luminosity ratios between the components (Table 5). An uncertainty of $15 \%$, correlated in $V$ and $I$, has been assumed for $l_{3}$.

For the determination of effective temperatures from the $(V-I)$ indices, we adopt an interstellar reddening of $E(B-V)=$ $0.15 \pm 0.02$, see Sect. 5.1, and assume $[\mathrm{Fe} / \mathrm{H}]=+0.40 \pm 0.10$, see Sect. 5.2. The calibration by Ramírez \& Meléndez (2005) then gives $5715 \pm 125 \mathrm{~K}$ and $4750 \pm 150 \mathrm{~K}$ for the primary and secondary components, respectively, whereas $5665 \pm 100 \mathrm{~K}$ and $4900 \pm 100 \mathrm{~K}$ are obtained from the recent calibration by Casagrande et al. (2006). From the empirical flux scale by Popper (1980), the bolometric correction (BC) scale by Flower (1996), and the $V$ flux ratio between the components, which is obtained to high precision from the light curve analyses (Table 5), we get a well constrained temperature difference of $765 \pm 15 \mathrm{~K}$, supporting the results from the Casagrande et al. calibration. We consequently adopt 5665 and $4900 \mathrm{~K}$ as our final $T_{\text {eff }}$ results.

As seen in Table 9 the synchronous rotational velocities of the components of V20 are small. Synthetic binary spectra based on these velocities and a resolution of 37000 qualitatively agree well with the observed spectra, but we have not attempted to determine the actual rotational velocities.

Eclipsing binaries are important primary distance indicators (Clausen 2004), and V20 provides a more direct determination for NGC 6791 than traditional main-sequence fitting. The two components yield nearly identical distances $(4000 \mathrm{pc}$ or $\left(V_{0}-M_{V}\right)=13$ m 00$)$, which have been established to a robust accuracy of 5\%, taking into account all error sources. For comparison, recently published results from main-sequence fitting, which show a spread significantly higher than the quoted errors, are: $12^{\mathrm{m}} 79(\mathrm{SBG} 03 ; E(B-V)=0.09$ and $[\mathrm{Fe} / \mathrm{H}]=0.3)$, 13. $07 \pm 0.04$ (Carney et al. 2005; $E(B-V)=0.14$ and $[\mathrm{Fe} / \mathrm{H}]=0.4)$, and $13 . \mathrm{m} 1 \pm 0 . \mathrm{m} 1(\mathrm{ATTM} 07 ; E(B-V)=0.155$ and $[\mathrm{Fe} / \mathrm{H}]=0.45)$.

\section{NGC 6791}

\subsection{The reddening of NGC 6791}

Anthony-Twarog et al. (2007) gave a thorough discussion of the reddening towards NGC 6791 based on $v b y C a H \beta$ photometry and a recalibration suitable for metal-rich stars, such as those found in this cluster. Over the years, since the first study of Kinman (1965), there has been significant disagreement over the reddening value. Here we adopt a value of $E(B-V)=0.15 \pm$ 0.02 for the cluster, giving most weight to the ATTM07 value which also agrees well with the value derived from the maps of Schlegel et al. (1998).

\subsection{Cluster metallicity}

As is the case for the reddening towards NGC 6791, the determination of its metallicity also has a long history. We shall not repeat it here but refer the reader to ATTM07 and Carretta et al. (2007) for a more comprehensive discussion. Based on the intense interest over the past few years, we shall adopt the 
Table 7. Spectroscopic elements for V20 determined from the two spectral regions applying different templates. Both double-lined and singlelined solutions are included. Epoch and orbital period were fixed at the values listed in Eq. (1), and a circular orbit was assumed. $K_{\mathrm{p}}$ and $K_{\mathrm{s}}$ are the velocity semi-amplitudes for primary and secondary component, respectively, $\gamma$ is the system velocity for V20, and $R V_{3}$ is the mean value of the radial velocity for the 3rd star. $\sigma$ is the standard error of one observation. All quantities are given in units of $\mathrm{km} \mathrm{s}^{-1}$. Spectra regions (R) are: $L=5100-5600 \AA$; $U=6100-6700 \AA$ with $\mathrm{H}_{\alpha}$ blocked out. Templates $(\mathrm{T})$ used are P (Primary): $T_{\text {eff }}=5600 \mathrm{~K}, \log (g)=4.2,[\mathrm{Fe} / \mathrm{H}]=+0.4 ; \mathrm{S}$ (Secondary): $T_{\text {eff }}=4800 \mathrm{~K}, \log (g)=4.6,[\mathrm{Fe} / \mathrm{H}]=+0.4 ; \mathrm{C}(3 \mathrm{rd}$ star $): T_{\text {eff }}=5000 \mathrm{~K}, \log (g)=4.5,[\mathrm{Fe} / \mathrm{H}]=+0.4$. The solutions marked by $*$ are based on velocities corrected by the results from the analyses of synthetic spectra; see text for details.

\begin{tabular}{llcccccc}
\hline \hline $\mathrm{R}$ & $\mathrm{T}$ & $K_{\mathrm{p}}$ & $K_{\mathrm{s}}$ & $\sigma_{\mathrm{p}}$ & $\sigma_{\mathrm{s}}$ & $\gamma$ & $R V_{3}$ \\
\hline $\mathrm{L}$ & $\mathrm{P}$ & $47.04 \pm 0.20$ & $61.22 \pm 0.20$ & 0.37 & 0.89 & $-46.49 \pm 0.12$ & $-44.43 \pm 0.61$ \\
$\mathrm{~L}^{*}$ & $\mathrm{P} *$ & $47.01 \pm 0.19$ & $61.31 \pm 0.19$ & 0.37 & 0.86 & $-46.49 \pm 0.12$ & $-44.43 \pm 0.61$ \\
$\mathrm{~L}$ & $\mathrm{~S}$ & $47.15 \pm 0.20$ & $61.13 \pm 0.20$ & 0.39 & 0.92 & $-46.46 \pm 0.12$ & $-44.52 \pm 0.57$ \\
$\mathrm{~L}$ & $\mathrm{C}$ & $47.13 \pm 0.20$ & $61.14 \pm 0.20$ & 0.38 & 0.89 & $-46.47 \pm 0.12$ & $-44.52 \pm 0.57$ \\
$\mathrm{U}$ & $\mathrm{P}$ & $47.19 \pm 0.11$ & $61.24 \pm 0.11$ & 0.35 & 0.43 & $-46.56 \pm 0.07$ & $-44.55 \pm 0.73$ \\
$\mathrm{U}^{*}$ & $\mathrm{P} *$ & $47.07 \pm 0.11$ & $61.18 \pm 0.11$ & 0.37 & 0.41 & $-46.50 \pm 0.07$ & $-44.50 \pm 0.73$ \\
$\mathrm{U}$ & $\mathrm{S}$ & $47.18 \pm 0.15$ & $61.16 \pm 0.15$ & 0.36 & 0.62 & $-46.54 \pm 0.09$ & $-44.49 \pm 0.71$ \\
$\mathrm{U}$ & $\mathrm{C}$ & $47.19 \pm 0.13$ & $61.15 \pm 0.14$ & 0.36 & 0.57 & $-46.54 \pm 0.08$ & $-44.49 \pm 0.70$ \\
$\mathrm{~L}$ & $\mathrm{P}$ & $46.98 \pm 0.08$ & & 0.27 & & $-46.71 \pm 0.07$ & \\
$\mathrm{~L} *$ & $\mathrm{P} *$ & $46.96 \pm 0.08$ & & 0.27 & & $-46.71 \pm 0.07$ & \\
$\mathrm{~L}$ & $\mathrm{~S}$ & $47.08 \pm 0.08$ & & 0.26 & & $-46.71 \pm 0.07$ & \\
$\mathrm{~L}$ & $\mathrm{C}$ & $47.06 \pm 0.08$ & & 0.26 & & $-46.71 \pm 0.07$ & \\
$\mathrm{U}$ & $\mathrm{P}$ & $47.17 \pm 0.10$ & & 0.33 & & $-46.63 \pm 0.09$ & \\
$\mathrm{U} *$ & $\mathrm{P} *$ & $47.04 \pm 0.10$ & & 0.33 & & $-46.63 \pm 0.09$ & \\
$\mathrm{U}$ & $\mathrm{S}$ & $47.17 \pm 0.11$ & & 0.35 & & $-46.62 \pm 0.09$ & \\
$\mathrm{U}$ & $\mathrm{C}$ & $47.17 \pm 0.10$ & & 0.34 & & $-46.62 \pm 0.09$ & \\
$\mathrm{~L}$ & $\mathrm{P}$ & & $61.16 \pm 0.25$ & & 0.82 & $-46.26 \pm 0.22$ & \\
$\mathrm{~L}$ & $\mathrm{~S}$ & & $61.07 \pm 0.26$ & & 0.84 & $-46.20 \pm 0.22$ & \\
$\mathrm{~L}$ & $\mathrm{C}$ & & $61.07 \pm 0.25$ & & 0.81 & $-46.22 \pm 0.21$ & \\
$\mathrm{U}$ & $\mathrm{P}$ & & $61.22 \pm 0.13$ & & 0.41 & $-46.50 \pm 0.11$ & \\
$\mathrm{U}$ & $\mathrm{S}$ & & $61.14 \pm 0.18$ & & 0.56 & $-46.48 \pm 0.16$ & \\
$\mathrm{U}$ & $\mathrm{C}$ & & $61.13 \pm 0.17$ & & 0.54 & $-46.47 \pm 0.14$ & \\
\hline
\end{tabular}

Table 8. Spectroscopic orbital solution for V20. $T$ is the time of central primary eclipse.

\begin{tabular}{lr}
\hline \hline Parameter & \multicolumn{1}{c}{ Value } \\
\hline Adjusted quantities: & \\
$K_{\mathrm{p}}\left(\mathrm{km} \mathrm{s}^{-1}\right)$ & $47.09 \pm 0.09$ \\
$K_{\mathrm{s}}\left(\mathrm{km} \mathrm{s}^{-1}\right)$ & $61.16 \pm 0.20$ \\
$\gamma\left(\mathrm{km} \mathrm{s}^{-1}\right)$ & $-46.63 \pm 0.13$ \\
Adopted quantities: & \\
$T$ (HJD-2 400000$)$ & 53151.6061 \\
$P($ days $)$ & 14.469918 \\
$e$ & 0.00 \\
Derived quantities: & \\
$M_{\mathrm{p}} \sin ^{3} i\left(M_{\odot}\right)$ & $1.074 \pm 0.008$ \\
$M_{\mathrm{s}} \sin ^{3} i\left(M_{\odot}\right)$ & $0.827 \pm 0.004$ \\
$a \sin i\left(R_{\odot}\right)$ & $30.946 \pm 0.063$ \\
Other quantities: & \\
$N_{\text {obs }}$ & 15 \\
Time span (days) & 377 \\
\hline
\end{tabular}

values determined spectroscopically. While a full consensus has not been reached on this subject, most recent determinations seem to agree on a value close to $[\mathrm{Fe} / \mathrm{H}]=+0.40$, which we adopt together with an uncertainty of \pm 0.10 dex.

We note that e.g. Carretta et al. report significant underabundances for $\mathrm{C}, \mathrm{N}$, and $\mathrm{O}$ of about -0.3 dex. Like Carraro et al. (2006) they find scaled-solar $\alpha$-element abundances.

\section{Comparison with theoretical models}

In the following, we compare the accurate dimensions obtained for V20, and the NGC 6791 CMDs, with properties of the VRSS (scaled-solar mixture) Victoria-Regina evolutionary tracks and isochrones (VandenBerg et al. 2006) ${ }^{4}$, the $Y^{2}$ (Yonsei-Yale) grids (Demarque et al. 2004) $)^{5}$, and the extensive material available from the BaSTI database (Pietrinferni et al. 2004) ${ }^{6}$. These recent models are all based on up-to-date input physics but differ somewhat with respect to e.g. core overshoot and diffusion (if included) treatment, He enrichment law, adopted solar mixture, and envelope convection calibration. Furthermore their associated colour transformations are different. We refer to Clausen et al. (2008) for further details, as well as to the original papers.

Throughout we have adopted scaled-solar mixture for the heavy elements, and we have selected the available models and isochrones which are closest to the adopted $[\mathrm{Fe} / \mathrm{H}]=+0.40 \pm$ 0.10 for the comparisons.

\subsection{V20}

For a given mass and $[\mathrm{Fe} / \mathrm{H}]$, the observable properties of models at a given age like radius, effective temperature, and luminosity depend on the adopted input physics, including treatment of core and envelope convection, diffusion etc., and the assumed $Y$, $Z$ relation. In order to constrain such free model parameters, accurate results from many binaries are needed; cluster members are particularly valuable. From the binary perspective, masses

\footnotetext{
${ }^{4}$ http://www1.cadc-ccda.hia-iha.nrc-cnrc.gc.ca/cvo/ community/VictoriaReginaModels/

5 http://www.astro.yale.edu/demarque/yystar.html

${ }^{6}$ http://www . te.astro.it/BASTI/index.php
} 

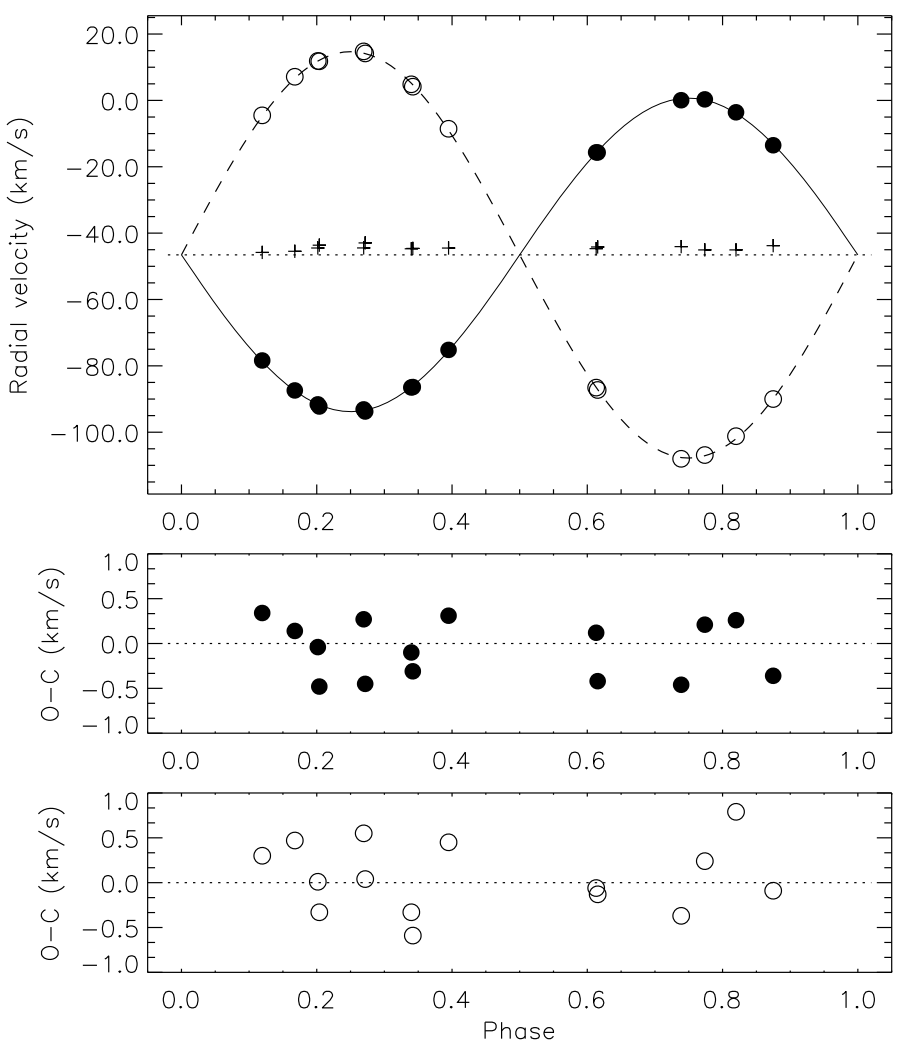

Fig. 8. Spectroscopic double-lined orbital solution for V20 obtained from the 6100-6700 $\AA$ region using the primary (P) template; see Table 7. Filled circle: primary; open circle: secondary. The horizontal dotted line (upper panel) represents the center-of-mass velocity of V20. The + signs are the measured velocities for the third light component. Phase 0.0 corresponds to central primary eclipse.

and radii are the most direct parameters available, free of any scale dependent calibrations. So, the $M-R$ diagram is well suited for isochrone tests, especially when the binary components are significantly different, as is the case for V20. In addition, the $T_{\text {eff }}-R$ plane allows tests of model temperatures, which for a given mass and $[\mathrm{Fe} / \mathrm{H}]$ depend on e.g. abundance mixture leading to $Z$, on $Y$, and on surface convection efficiency. Adopting the usual assumption of coeval formation of the components from the same raw material, identical ages must be reached in these two planes, as well as in the $M-\log (L)$ plane.

From the $M-R$ comparisons shown in Figs. 9-11 we derive the component ages listed in Table 10. As seen, mass and radius uncertainties translate to age uncertainties of about $0.3 \mathrm{Gyr}$ (primary) and 0.9 Gyr (secondary), respectively, for a given adopted composition. However, at $[\mathrm{Fe} / \mathrm{H}]=0.40$, the ages resulting from the three grids range between 7.6 and 9.0 Gyr for the primary and between 6.2 and 8.6 for the secondary. Within uncertainties, the Victoria-Regina and BaSTI isochrones predict identical ages for the two components, although the age for the secondary is systematically lower than that of the primary. The $Y^{2}$ grids clearly predict different ages, again with lower values for the secondary. Age uncertainties due to an $[\mathrm{Fe} / \mathrm{H}]$ uncertainty of 0.1 dex are comparable to those coming from masses and radii.

We note that this trend of lower ages for the less massive star is opposite to what has been found for several field G-type eclipsing binaries; see e.g. Popper (1997), Clausen et al. (1999), Torres et al. (2006), and Clausen et al. (in prep.). In these cases, the seemingly higher ages may, although not yet firmly proved, be due to surface activity and correlated less efficient convection
Table 9. Astrophysical data for V20. $T_{\text {eff } \odot}=5780 \mathrm{~K}, B . C \cdot \odot=-0.08$, and $M_{\mathrm{bol} \odot}=4.74$ has been assumed. We have adopted $E(B-V)=$ $0.15 \pm 0.02, E(V-I)=1.3 \times E(B-V)$, and $A_{V}=3.1 \times E(B-V) \cdot v_{\text {sync }}$ is the equatorial velocity for synchronous rotation.

\begin{tabular}{lrr}
\hline \hline & Primary & Secondary \\
\hline Absolute dimensions: & & \\
$M / M_{\odot}$ & $1.074 \pm 0.008$ & $0.827 \pm 0.004$ \\
$R / R_{\odot}$ & $1.399 \pm 0.016$ & $0.768 \pm 0.006$ \\
$\log g(\mathrm{cgs})$ & $4.178 \pm 0.010$ & $4.586 \pm 0.008$ \\
& & \\
$v_{\text {sync }}\left(\mathrm{km} \mathrm{s}^{-1}\right)$ & $4.9 \pm 0.1$ & $2.7 \pm 0.1$ \\
Photometric data: & & \\
$V$ & $17.642 \pm 0.028$ & $19.867 \pm 0.041$ \\
$I$ & $16.729 \pm 0.033$ & $18.639 \pm 0.046$ \\
$(V-I)$ & $0.914 \pm 0.008$ & $1.228 \pm 0.044$ \\
$V_{0}$ & $17.177 \pm 0.068$ & $19.400 \pm 0.074$ \\
$(V-I)_{0}$ & $0.719 \pm 0.027$ & $1.031 \pm 0.051$ \\
$T_{\text {eff }}(\mathrm{K})$ & & \\
$M_{\text {bol }}$ & $5665 \pm 100$ & $4900 \pm 100$ \\
$\log L / L_{\odot}$ & $4.10 \pm 0.08$ & $6.03 \pm 0.09$ \\
$B . C$. & $0.26 \pm 0.03$ & $-0.52 \pm 0.04$ \\
$M_{V}$ & -0.10 & -0.35 \\
$V-M_{V}$ & $4.20 \pm 0.08$ & $6.38 \pm 0.09$ \\
$V_{0}-M_{V}$ & & \\
Distance $(\mathrm{pc})$ & $13.44 \pm 0.09$ & $13.48 \pm 0.10$ \\
\hline & $12.98 \pm 0.11$ & $13.02 \pm 0.12$ \\
& $3940 \pm 200$ & $4015 \pm 225$ \\
\hline
\end{tabular}

Table 10. Ages for the componets of V20 as determined from $M-R$ isochrones calculated for different models and chemical compositions. For each model, the first line represent the available models closest to the observed $[\mathrm{Fe} / \mathrm{H}]$. All models include core overshoot; BaSTI standard and core overshoot models yield nearly identical results. Uncertainties due to mass and radius errors are about 0.3 Gyr (primary) and 0.9 Gyr (secondary).

\begin{tabular}{llll}
\hline \hline Model & {$[\mathrm{Fe} / \mathrm{H}]$} & Primary & Secondary \\
\hline VRSS & 0.37 & 7.7 & 7.2 \\
& 0.23 & 7.8 & 7.0 \\
$Y^{2}$ & 0.49 & 7.3 & 6.8 \\
& 0.40 & 8.2 & 6.2 \\
& 0.30 & 8.3 & 6.2 \\
BaSTI & 0.50 & 7.8 & 6.2 \\
& 0.395 & 9.0 & 8.6 \\
& 0.254 & 8.5 & 7.6 \\
\hline
\end{tabular}

at the less massive components, which result in larger radius and lower effective temperature (but nearly unchanged luminosity).

In Fig. 12, we compare the Victoria-Regina, BaSTI, and $Y^{2}$ isochrones fitting the primary component. The first two are nearly identical, but for an age difference of as much as $1.3 \mathrm{Gyr}$, whereas the shape of the $Y^{2}$ isochrone, at an age approximately midway between the two, is significantly different.

Turning to the $T_{\text {eff }}-R$ plane shown in Figs. 13-15, it is seen that the Victoria-Regina tracks fit both components within errors. They are very slightly hotter than observed, and as seen in Fig. 13 this is also the case for tracks calculated for a higher $[\mathrm{Fe} / \mathrm{H}]=0.49$, which actually fall close to the $[\mathrm{Fe} / \mathrm{H}]=0.37$ tracks. The $Y^{2}$ tracks are cooler than observed, and here tracks for a 0.1 dex lower $[\mathrm{Fe} / \mathrm{H}]$ agree better. The BaSTI tracks, both standard and with core overshoot, fit the components of V20 perfectly well. 


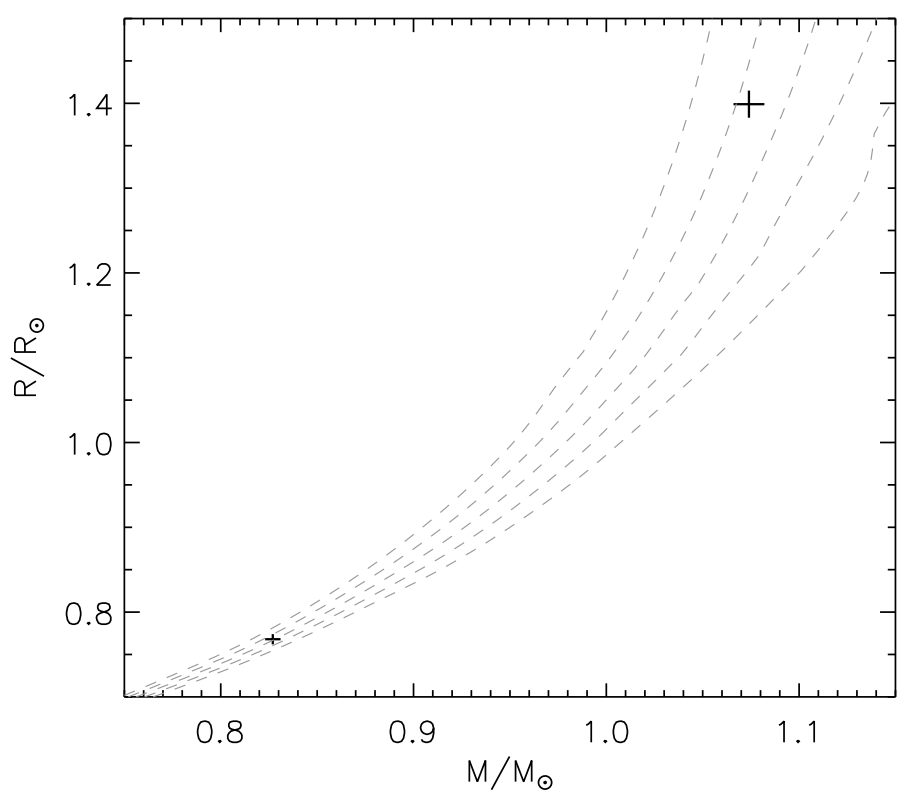

Fig. 9. V20 compared to Victoria-Regina VRSS models for $(X, Y$, $Z)=(0.63656,0.32344,0.04000)$, equivalent to $[\mathrm{Fe} / \mathrm{H}]=+0.37$ for $[\alpha / \mathrm{Fe}]=0.00$. Isochrones from 5.0 to 9.0 Gyr (step 1.0 Gyr) are shown.

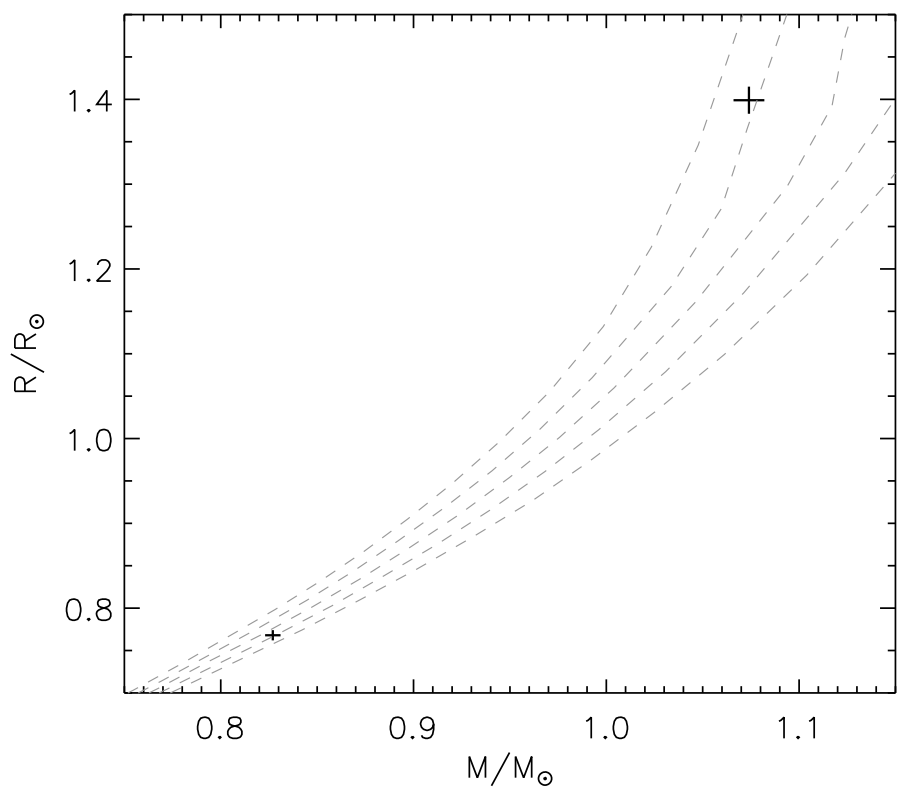

Fig. 10. V20 compared to $Y^{2}$ models for $(X, Y, Z)=(0.6467,0.3122$, 0.0411 ), equivalent to $[\mathrm{Fe} / \mathrm{H}]=+0.40$ for $[\alpha / \mathrm{Fe}]=0.00$. Isochrones from 5.0 to $9.0 \mathrm{Gyr}$ (step 1.0 Gyr) are shown.

In conclusion, both the the Victoria-Regina and the BaSTI models represent V20 well, but at ages which differ by about 1.3 Gyr. This is significantly higher than the precision of about $0.5 \mathrm{Gyr}$, which can be reached from the available mass, radius, and abundance information. The cause(s) for this difference is not clear but could perhaps be related to the fact, that the Victoria-Regina models apply different $Y, Z$ relations and core overshoot treatment. Direct comparisons between VictoriaRegina and BaSTI tracks and isochrones are shown in Figs. 16 and 17.

Additional NGC 6791 binaries with component(s) between 0.85 and $1.0 M_{\odot}$ would add further constraints on the models, and would e.g. clearly reveal if the isochrone shape is correct. Also, similar comparisons for younger clusters would

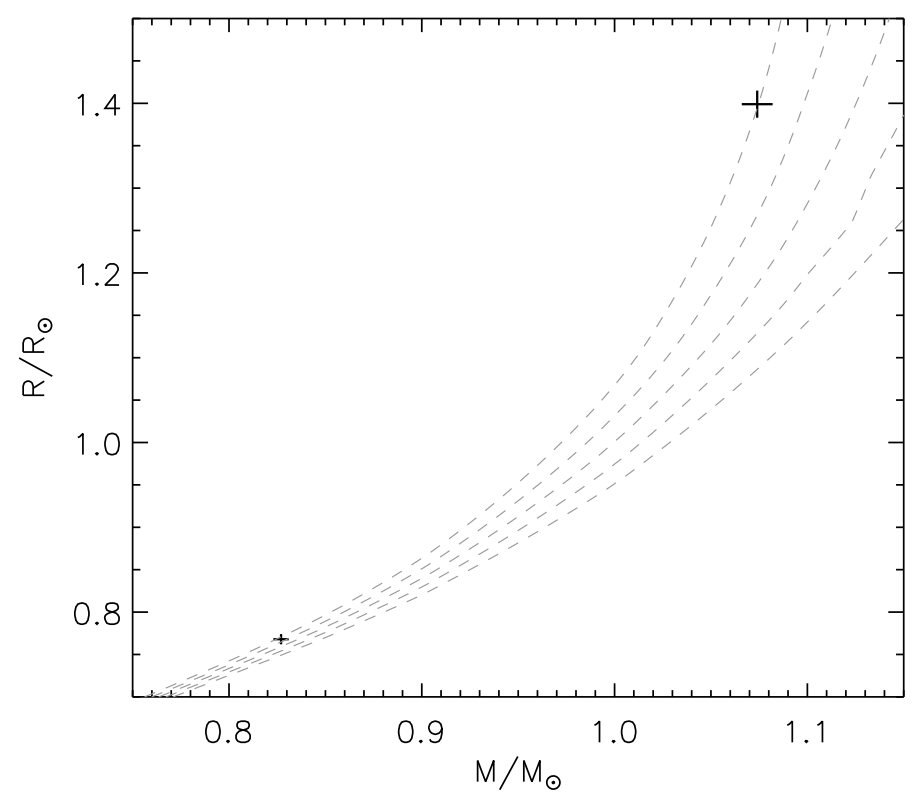

Fig. 11. V20 compared to BaSTI models with overshooting for $(X$, $Y, Z)=(0.657,0.303,0.0400)$, equivalent to $[\mathrm{Fe} / \mathrm{H}]=+0.395$ for $[\alpha / \mathrm{Fe}]=0.00$. Isochrones from 5.0 to $9.0 \mathrm{Gyr}$ (step 1.0 Gyr) are shown.

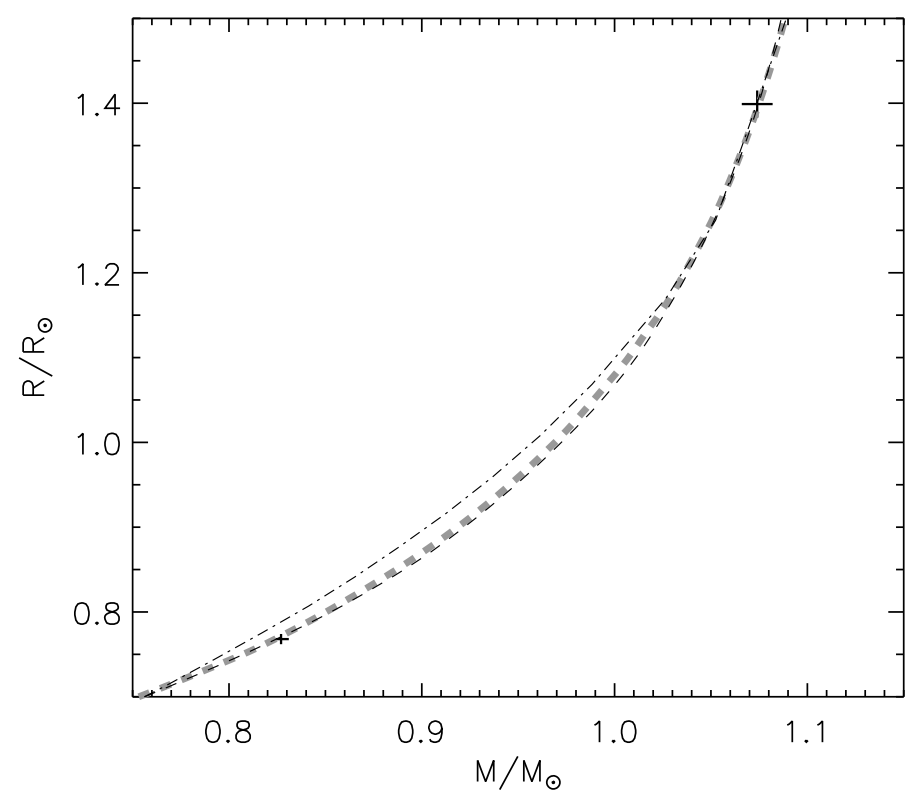

Fig. 12. V20 compared to the VRSS (7.7 Gyr, dashed thick gray), $Y^{2}$ (8.2 Gyr, dashed-dot black), and BaSTI (9.0 Gyr, dashed black) isochrones which fit the primary component at the observed $[\mathrm{Fe} / \mathrm{H}]$; see Table 10.

be valuable. We note that for 1-2 Gyr field F-type binaries with component masses in the 1.1-1.4 $M_{\odot}$ range, Clausen et al. (2008) recently found that the Victoria-Regina models are superior to the BaSTI models.

\subsection{NGC 6791 colour-magnitude diagrams}

In light of the results obtained from V20 in the previous section, it is interesting to investigate what extra information can be gained from a comparison of the isochrones to the CMD of the cluster. There is an abundant literature on age determination of old open - and globular clusters using "isochrone-fitting". The method is well described and so are the problems involved in the 


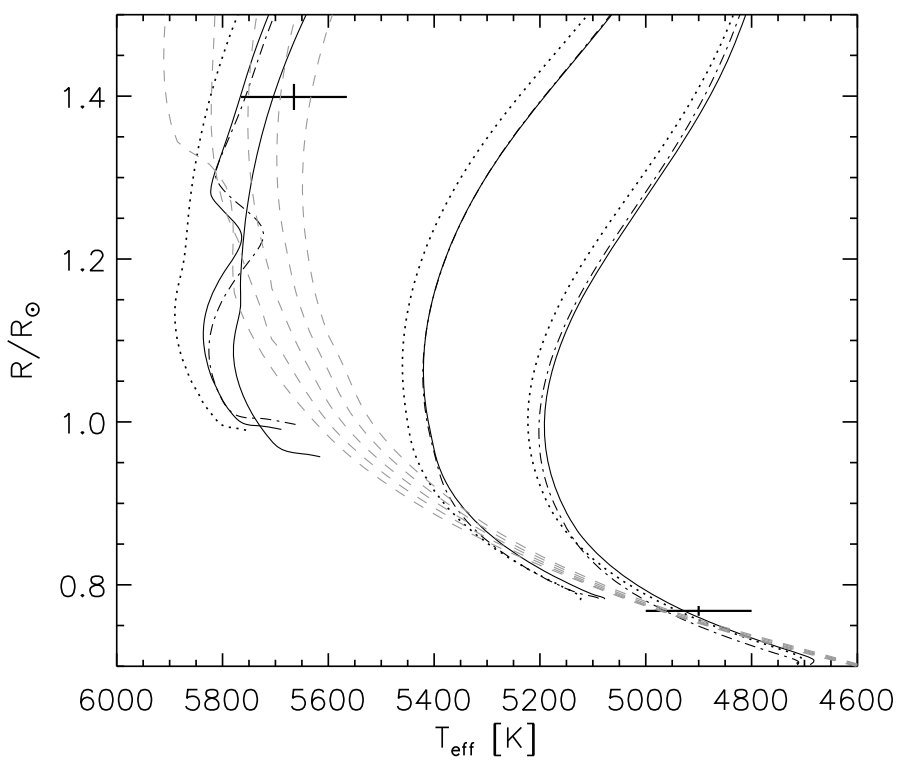

Fig. 13. V20 compared to Victoria-Regina VRSS models for $(X, Y$, $Z)=(0.63656,0.32344,0.04000)$, equivalent to $[\mathrm{Fe} / \mathrm{H}]=+0.37$ for $[\alpha / \mathrm{Fe}]=0.00$. Tracks for $0.8,0.9,1.073$, and $1.1 M_{\odot}$ (full drawn) and isochrones from 5.0 to $9.0 \mathrm{Gyr}$ (dashed, step $1.0 \mathrm{Gyr}$ ) are shown. For comparison, tracks for $0.8,0.9$, and $1.1 M_{\odot}$ for $(X, Y, Z)=(0.66856$, $0.30144,0.03000$ ), equivalent to $[\mathrm{Fe} / \mathrm{H}]=+0.23$ for $[\alpha / \mathrm{Fe}]=0.00$ (dotted), and for $(X, Y, Z)=(0.60456,0.34544,0.05000)$, equivalent to $[\mathrm{Fe} / \mathrm{H}]=+0.49$ for $[\alpha / \mathrm{Fe}]=0.00$ (dot-dash), are included.

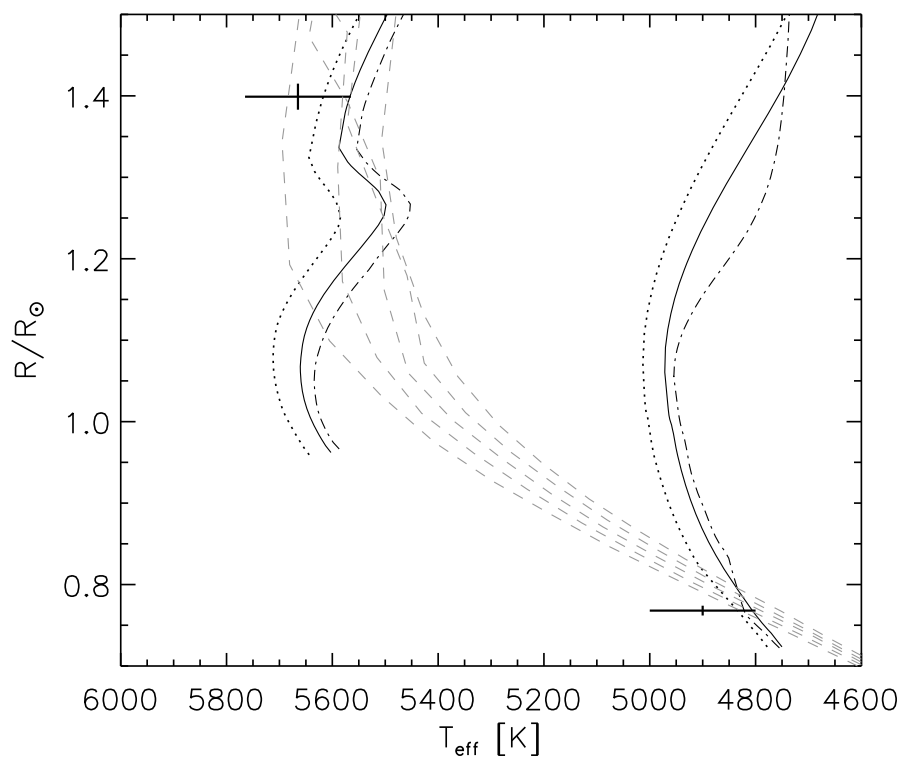

Fig. 14. V20 compared to $Y^{2}$ models for $(X, Y, Z)=(0.6467,0.3122$, $0.0411)$, equivalent to $[\mathrm{Fe} / \mathrm{H}]=+0.40$ for $[\alpha / \mathrm{Fe}]=0.0$. Tracks for the component masses (1.074 and $0.827 M_{\odot}$, full drawn) and isochrones from 5.0 to $9.0 \mathrm{Gyr}$ (dashed, step $1.0 \mathrm{Gyr}$ ) are shown. To illustrate the effect of the abundance uncertainty, tracks for $[\mathrm{Fe} / \mathrm{H}]=+0.30$ and $[\alpha / \mathrm{Fe}]=0.00$ (dotted), corresponding to $(X, Y, Z)=(0.6686,0.2976$, 0.0338 ), and $[\mathrm{Fe} / \mathrm{H}]=+0.50$ and $[\alpha / \mathrm{Fe}]=0.00$ (dash-dot), corresponding to $(X, Y, Z)=(0.6200,0.3300,0.0500)$, are included.

process. Observationally it is necessary to obtain accurate determinations of reddening, distance and cluster abundance, and subsequently match theoretical models shifted in colour and luminosity to the observed CMD. The theoretical models must be transformed to the observational plane (colour and magnitude) in order to allow the comparison. It is well known that for old

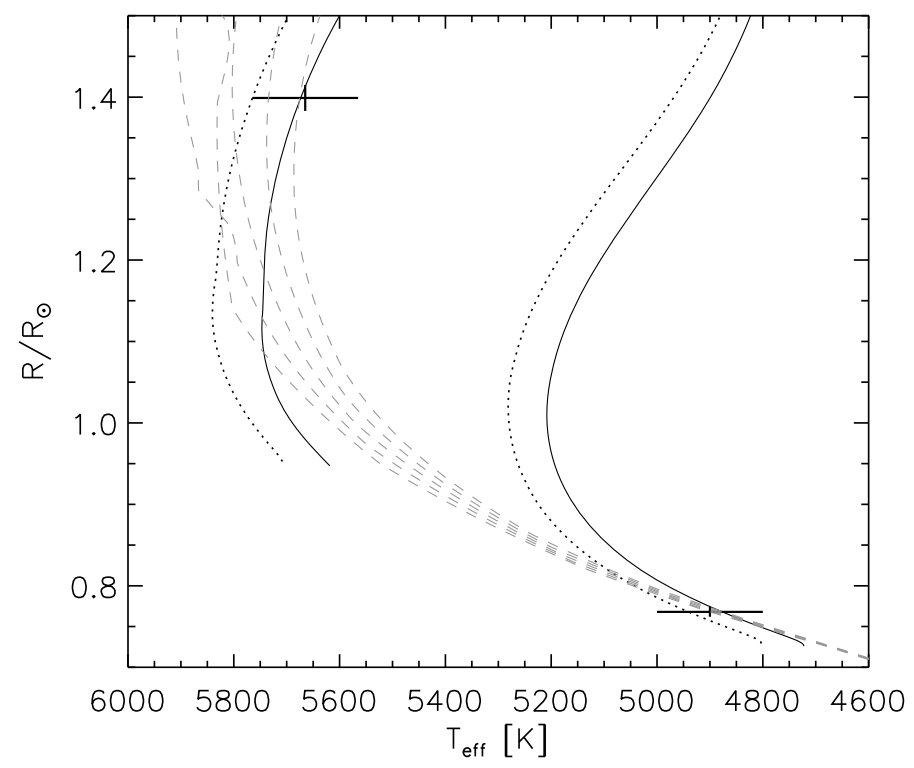

Fig. 15. V20 compared to BaSTI models with overshooting for $(X$, $Y, Z)=(0.657,0.303,0.0400)$, equivalent to $[\mathrm{Fe} / \mathrm{H}]=+0.395$ for $[\alpha / \mathrm{Fe}]=0.00$. Tracks for the component masses $\left(1.074\right.$ and $0.827 M_{\odot}$, full drawn) and isochrones from 5.0 to $9.0 \mathrm{Gyr}$ (dashed, step $1.0 \mathrm{Gyr}$ ) are shown. For comparison, tracks (dotted) for $(X, Y, Z)=(0.682,0.288$, 0.0300 ), equivalent to $[\mathrm{Fe} / \mathrm{H}]=+0.254$ for $[\alpha / \mathrm{Fe}]=0.00$, are included.

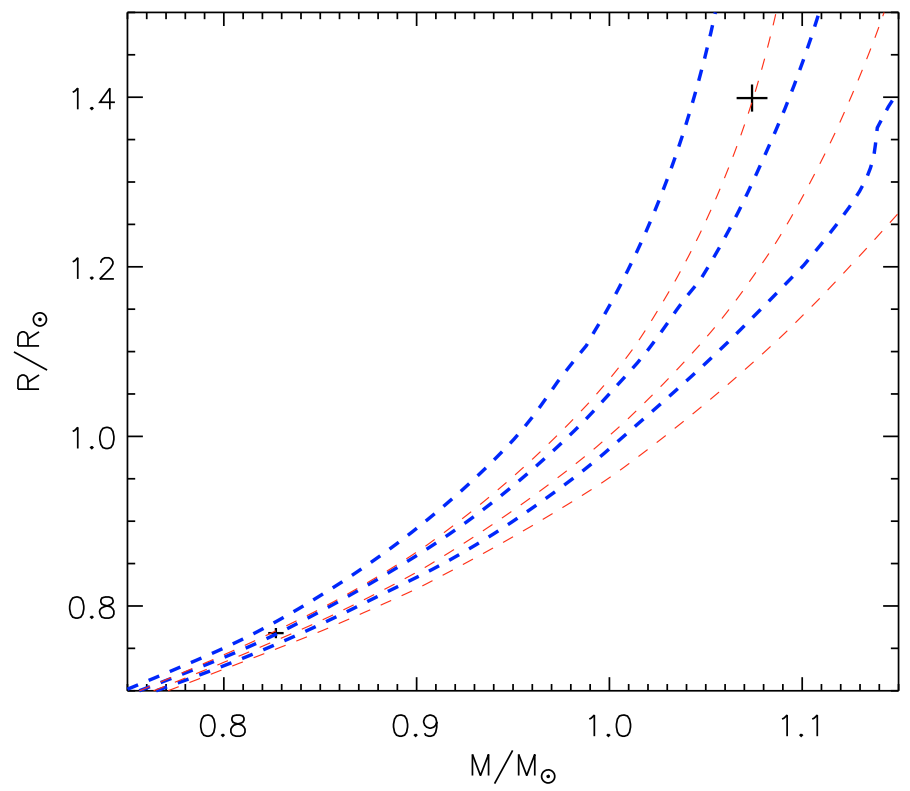

Fig. 16. Comparison between VRSS (thick blue) and BaSTI (thin red) isochrones at 5, 7, and $9 \mathrm{Gyr}$ for compositions close to the observed $[\mathrm{Fe} / \mathrm{H}]$; see Table 10 . The components of V20 are included for comparison.

clusters, the isochrone shapes only change slowly with time, and thus age-determinations based on isochrone shapes are not very sensitive. Furthermore, the turnoff luminosity and colour also changes slowly with time. In the best cases, the accuracies obtained for reddening and distance modulus of clusters is of the order 0.01 and 0.1 , respectively (Pasquini et al. 2008). For an 8 Gyr old cluster such errors translate into an uncertainty in age (from the turnoff position) of $\sim 0.5 \mathrm{Gyr}$ and $\sim 1.0 \mathrm{Gyr}$, respectively (estimated from VRSS isochrones and for $[\mathrm{Fe} / \mathrm{H}]=0.4$ ). Thus, to obtain age estimates better than $1 \mathrm{Gyr}$, very precise 


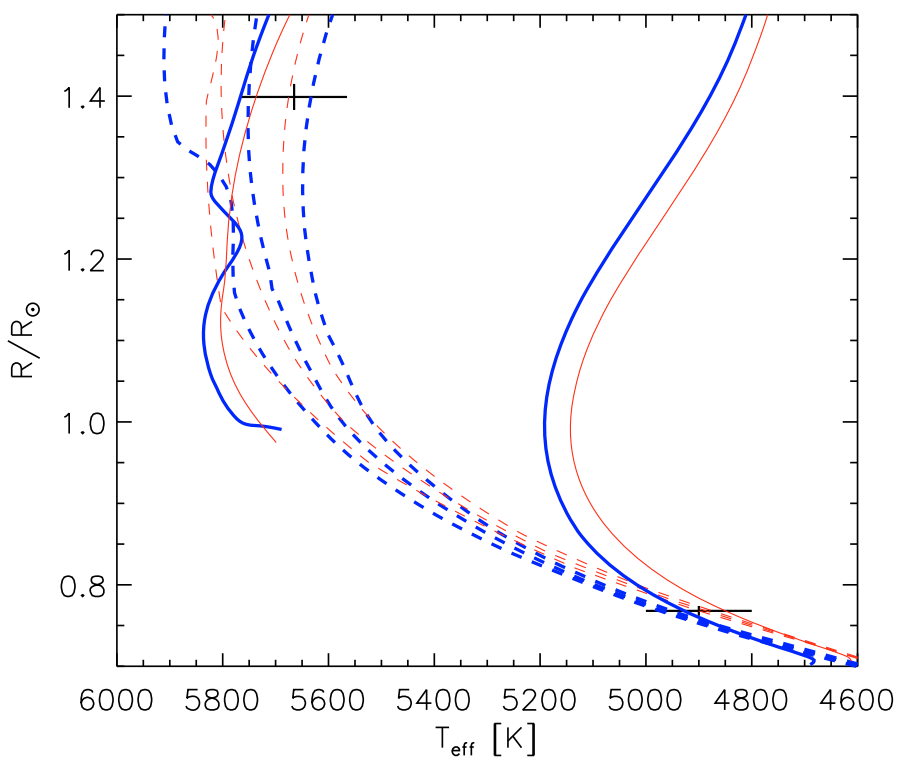

Fig. 17. Comparison between VRSS (thick blue) and BaSTI (thin red) 1.1 and $0.8 M_{\odot}$ tracks (full drawn) and 5, 7, and 9 Gyr isochrones (dashed). for compositions close to the observed [Fe/H]; see Table 10. The components of V20 are included for comparison

values for reddening and distance must be obtained - in addition to this the models also show a non-negligible dependence on the adopted metallicity.

VandenBerg \& Stetson (2004), Fig. 7, present a systematic investigation of what effect plausible changes in metallicity and distance modulus (at fixed reddening) has on the derived age for NGC 188, which is 2 Gyr younger than NGC 6791. Their exercise shows that ages between 5.9 and $8.1 \mathrm{Gyr}$ are compatible with the cluster CMD corresponding to $\sim 30 \%$ of the cluster age.

To confront the theoretical models with the observations we show, in Fig. 18, the CMD for NGC 6791 in the $(B-V, V),(V-$ $I, V)$ and $(B-I, V)$ planes with the preferred age for each of the three isochrone sets (using the reddening and distance from Table 9). We employ the models closest to $[\mathrm{Fe} / \mathrm{H}]=+0.40$ throughout. It is evident that the isochrones are not far from matching the CMD in all colour planes, although the $Y^{2}$ models are somewhat too red compared to the VRSS and BaSTI models. From inspection of the figure, it is clear that offsets within 0 . 02 in reddening and 0.10 in distance-modulus would bring the models into agreement with the cluster turnoff region. We thus conclude that for the three age values, the isochrones in the CMD are not in direct conflict with the observations, except perhaps for the $(V-I)$ and $(B-I)$ colours of the $Y^{2}$ models. By simple experimenting with VRSS models it is also clear that with (plausible) modifications to the adopted reddening and distance modulus, it is posible to match the turnoff region for the cluster for ages spanning at least the 6-9 Gyr range; the same conclusion would also hold for BaSTI or $Y^{2}$ models.

Given the current uncertainties in the derivation of cluster reddening, distance modulus, and metallicity, it is the opinion of the authors that we can only gain limited information on cluster absolute ages from their colour-magnitude diagrams. From the results presented here, V20 provide much tighter constraints on the age of NGC 6791 than isochrone-fitting to the colourmagnitude diagram. In fact, it would be necessary to know the distance-modulus and intrinsic colour of the cluster turnoff to an accuracy better than 0.05 and 0.01 , respectively to match the 0.5 Gyr age precision.
Unfortunately, we are faced with the situation that even in the $M-R$ diagram, the models give very discrepant ages with differences of $15-20 \%$ (see Fig. 9). Until this situation is clarified, it is the models which limit the precision with which stellar ages can be obtained. We speculate that in the case of NGC 6791 the discrepancy could be due to difficulties in the description of convective energy transport.

\section{Summary and conclusions}

In this paper we have presented extensive photometric and spectroscopic observations of the detached eclipsing binary V20 in the old open cluster NGC 6791, and determined precise masses and radii for its primary and secondary components. The errors in masses and radii are not subject to the usual problems of determining the cluster reddening, metallicity and distance associated with cluster studies. This has allowed a determination of the cluster age with an uncertainty of only $0.5 \mathrm{Gyr}$ (errors due to mass, radius and metallicity), by comparing the location of the primary component to isochrones in the mass-radius plane. However, the absolute age(s) for the cluster derived from the Victoria-Regina, BaSTI and $Y^{2}$ isochrones differ by up to $1.3 \mathrm{Gyr}$, and yet the two extreme isochrones overlap almost perfectly in a $M-R$ diagram (Fig. 12). Until these discrepancies are resolved, this prevents us from drawing a firm conclusion on the true value for the cluster age.

The error in the age due to the uncertainty in the mass and radius is only 0.3 Gyr for a single isochrone set. This is $\sim 3$ times better than what is typically obtained using isochrone-fitting using CMDs.

By combining the stellar radii with a temperatures established from the photometry, we determined the cluster distance to be $4 \mathrm{kpc}$, with good agreement between the value from each binary component. This was used to compare the cluster CMD to isochrones, for an adopted reddening of $E(B-V)=0.15$. We found that for the derived distance and age, the three isochrone sets match the observed CMD reasonably well - however the obtainable age precision is not nearly as good as when using the binary components in a mass-radius diagram.

Our main conclusion is that with the needed precision of reddening, metallicity and distance estimates, accurate ages of clusters, based on "isochrone-fitting", will be very difficult to obtain until GAIA can provide high accuracy parallaxes.

In order to improve on cluster age-determinations, many more eclipsing systems in clusters should be investigated (Kaluzny et al. 2006). It would be particularly interesting to study clusters with more than one detached system (see e.g. Southworth et al. 2004a), as this would provide more constraints on the shapes of the isochrones in the $M-R$ diagram at fixed age. A set of well studied clusters with good CMDs and accurate masses and radii for several detached eclipsing systems would provide an excellent calibration set against which stellar models can be tested to high accuracy. By extending such studies to globular clusters detached eclipsing binaries could ultimately provide the strongest constraints on their ages. We have shown in this study that using $8 \mathrm{~m}$-class telescopes it is feasible to work at the required level of accuracy at $V=20$ thus making the turnoff stars in the nearest globular clusters, and most open clusters, available.

Finally, it is worthwhile to note, that with further observations of V20, it will be possible to reduce the size of the errorbars for both the mass and radius determination and thus provide even tighter constraints (for a given set of models) on the age of NGC 6791. With currently existing instrumentation it should be 


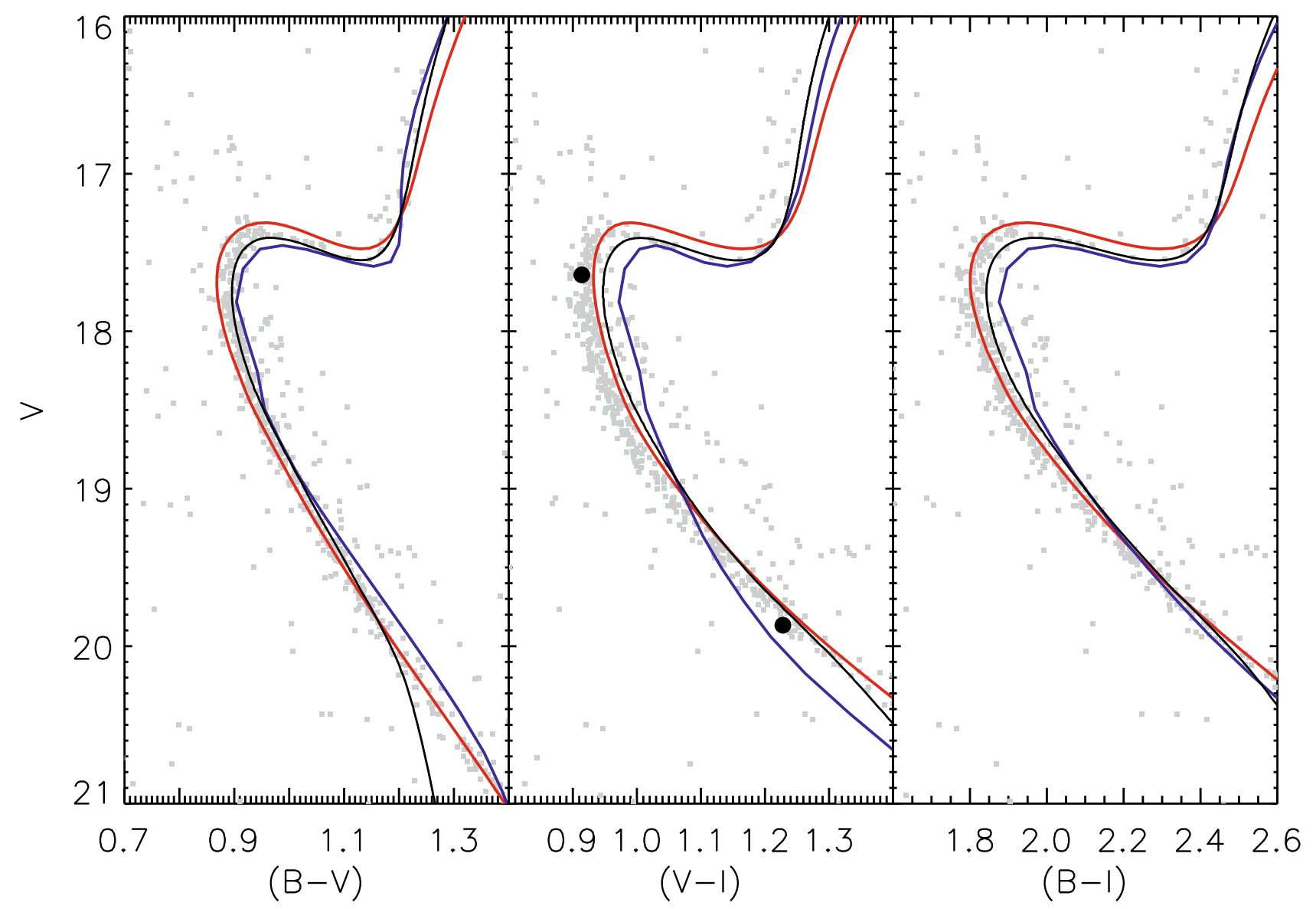

Fig. 18. Isochrones for ages 7.7 (VRSS, red), 8.2 ( $Y^{2}$, blue), and 9.0 Gyr (BaSTI, black) overplotted on the various CMDs of NGC 6791 (SBG03 photometry, plotted at light grey squares). In all plots we have adopted $E(B-V)=0.15$ and $(m-M)_{V}=13.46$ to transpose the isochrones to the observational plane (see Table 9). In the middle panel, the location of the primary and secondary component of V20 are shown as filled black circles. The models are the same as those used in Fig. 12. On black and white printers the models are hard to distinguish, but at the cluster turnoff the VRSS models are always the bluest and the $Y^{2}$ models always the reddest.

possible to reduce the errors in mass and radius to below $0.5 \%$ for both the primary and secondary components.

Acknowledgements. We thank H. Bruntt for making his bssynth software available and J. Southworth for access to JKTEBOP. P. Stetson is thanked for sharing his excellent photometry software with us. The project "Stars: Central engines of the evolution of the Universe", carried out at Aarhus University and the University of Copenhagen, is supported by the Danish National Science Research Council. F.G. acknowledges financial support from the Carlsberg Foundation, the Danish AsteroSeismology Centre at Aarhus University, and Instrumentcenter for Dansk Astronomi. The following internet-based resources were used in research for this paper: the NASA Astrophysics Data System; the SIMBAD database and the ViziR service operated by CDS, Strasbourg, France; the ar $\chi$ iv scientific paper preprint service operated by Cornell University.

\section{References}

Anthony-Twarog, B. J., Twarog, B. A., \& Mayer, L. 2007, AJ, 133, 1585 (ATTM07)

Bedin, L. R., Salaris, M., Piotto, G., et al. 2005, ApJ, 624, L45

Bedin, L. R., King, I. R., Anderson, J., et al. 2008, ApJ, 678, 1279

Bruntt, H., Grundahl, F., Tingley, B., et al. 2003, A\&A, 410, 323

Bruntt, H., Southworth, J., Torres, G., et al. 2006, A\&A, 456, 651

Carney, B. W., Lee, J.-W., \& Dodson, B. 2005, AJ, 129, 656

Carraro, G., Villanova, S., Demarque, P., et al. 2006, ApJ, 643, 1151

Carretta, E., Bragaglia, A., \& Gratton, R. G. 2007, A\&A, 473, 129

Casagrande, L., Portinari, L., \& Flynn, C. 2006, MNRAS, 373, 13

Claret, A. 2000, A\&A, 363, 1081
Clausen, J. V. 2004, New Ast. Rev., 48, 679

Clausen, J. V., Baraffe, I., Claret, A., \& VandenBerg, D. B. 1999, in Theory and Tests of Convection in Stellar Structure, ed. A. Giménez, E. F. Guinan, \& B. Montesinos, ASP Conf. Ser., 173, 265

Clausen, J. V., Torres, G., Bruntt, H., et al. 2008 A\&A, 487, 1095

de Marchi, F., Poretti, E., Montalto, M., et al. 2007, A\&A, 471, 515

Dekker, H., D’Odorico, S., Kaufer, A., Delabre, B., \& Kotzlowski, H. 2000, Proc. SPIE, 4008, 534

Demarque, P., Woo, J.-H., Kim, Y.-C., \& Yi, S. K. 2004, ApJS, 155, 667

Etzel P. B. 1981, in Photometric and Spectroscopic Binary Systems, ed. E. B. Carling, \& Z. Kopal (Dordrecht: Reidel Publishing Company)

Etzel, P. B. 2004, SBOP: Spectroscopic Binary Orbit Program, San Diego State University

Flower, P. J. 1996, ApJ, 469, 355

Grevesse, N., \& Noels, A. 1993, Phys. Scr. T, 47, 133

Grevesse, N., Noels, A., \& Sauval, A. J. 1996, in Cosmic Abundances, ed. S. S.

Holt, \& G. Sonneborn (San Francisco: ASP), 117

Van Hamme, W. 1993, AJ, 106, 2096

Heiter, U., Kupka, F., van't Menneret, C., et al. 2002, A\&A, 392, 619

Kaluzny, J., Pych, W., Rucinski, S. M., \& Thompson, I. B 2006, AcA, 56, 237

Kim, Y.-C., Demarque, P., Yi, S. K., \& Alexander, D. R. 2002, ApJS, 143, 499

Kinman, T. D. 1965, ApJ, 142, 655

Korn, A. J., Grundahl, F., Richard, O., et al. 2006, Nature, 442, 657

Kwee, K. K., \& van Woerden, H. 1956, BAN, 12, 327

Lacy, C. H. S., Torres, G., \& Claret, A. 2008, AJ, 135, 1757

Landsman, W., Bohlin, R. C., Neff, S. G., et al. 1998, AJ, 116, 789

Liebert, J., Saffer, R. A., \& Green, E. M. 1994, AJ, 107, 1408

Martynov, D. Ya. 1973, in Eclipsing Variable Stars, ed. V. P. Tsesevich, Israel Program for Scientific Translation, Jerusalem

Mochejska, B. J., Stanek, K. Z., Sasselov, D. D., \& \& Szentgyorgti, A. H. 2002, AJ, 123, 3460 
Mochejska, B. J., Stanek, K .Z., Sasselov, D. D., et al. 2005, AJ, 129, 2856 Montalto, M., Piotto, G., Desidera, S., et al. 2007, A\&A, 470, 1137

Nelson, B., \& Davis, W. 1972, ApJ, 174, 617

Origlia, L., Valenti, E., Rich, R. M., \& Ferraro, F. R. 2006, ApJ, 646, 499

Pasquini, L., Biazzo, K., Bonifacio, P., Randich, S., \& Bedin, L. 2008, A\&A, 489,677

Pietrinferni, A., Cassisi, S., \& Salaris, M. 2004, Mem. S.A.It., 75, 170

Popper, D. M. 1980, ARA\&A, 18, 115

Popper, D. M. 1997, AJ, 114, 1195

Popper, D. M., \& Etzel, P. B. 1981, AJ, 86, 102

Press, W. H., Flannery, B. P., Teukolsky, S. A., \& Vetterling, W. T. 1992,

Numerical Recepies in Fortran, 77, 2nd edn. (Cambridge: Cambridge Univ. Press)

Ramírez, I., \& Meléndez, J. 2005, AJ, 626, 465

Rucinski, S. M. 1999, in IAU Coll. 170, Precise Stellar Radial Velocities, ed. J. B. Hearnshaw, \& C. D. Scarfe, ASP Conf. Ser., 185, 82

Rucinski, S. M. 2002, AJ, 124, 1746

Rucinski, S. M. 2004, in IAU Symp. 215, Stellar Rotation, ed. A. Maeder, \& P. Eenens, ASP Conf. Ser., 17
Rucinski, S. M., Kaluzny, J., \& Hilditch, R. W. 1996, MNRAS, 282, 70 Schlegel, D. J., Finkbeiner, D. P., \& Davis, M. 1998, ApJ 500, 525

Southworth, J., Maxted, P. F. L., \& Smalley, B. 2004a, MNRAS, 349, 547

Southworth, J., Maxted, P. F. L., \& Smalley, B. 2004b, MNRAS, 351, 1277

Southworth, J., Zucker, S., Maxted, P. F. L., \& Smalley, B. 2004c, MNRAS, 355, 986

Southworth, J., Bruntt, H., \& Buzasi, D. L. 2007, A\&A, 467, 1215

Stetson, P. B. 1987, PASP, 99, 191

Stetson, P. B. 1990, PASP, 102, 932

Stetson, P. B. 1994, PASP, 106, 250

Stetson, P. B., Bruntt, H., \& Grundahl, F. 2003, PASP, 115, 413 (SBG03)

Torres, G., Lacy, C. H., Marschall, L. A., Sheets, H. A., \& Mader, J. A. 2006, AJ, 640, 1018

Tripicco, M. J., Bell, R. A., Dorman, B., \& Hufnagel, B. 1995, AJ, 109, 1697

VandenBerg, D. A., Swenson, F. J., Rogers, F. J., Iglesias, C. A., \& Alexander, D. R. 2000, ApJ, 532, 430

Valenti, J., \& Piskunov, N. 1996, A\&AS, 118, 595

VandenBerg, D. A., \& Stetson, P. B. 2004, PASP, 116, 997

VandenBerg, D. A., Bergbusch, P. A., \& Dowler, P. D. 2006 ApJS, 162, 375 